\title{
Large Deviations for Re-sequencing Buffer Size
}

\author{
by \\ Yanfei Gao \\ A thesis submitted to the Faculty of Graduate Studies and Research \\ in partial fulfillment of the requirements for the degree of

\section{Master of Science} \\ Ottawa-Carleton Institute for Mathematics and Statistics \\ Carleton University \\ 1125 Colonel By Drive, Ottawa, Ontario
}

Canada K1S 5B6

July, 2008

(C)Yanfei Gao 2008 


$\begin{array}{ll}\begin{array}{l}\text { Library and } \\ \text { Archives Canada }\end{array} & \begin{array}{l}\text { Bibliothèque et } \\ \text { Archives Canada }\end{array} \\ \begin{array}{l}\text { Published Heritage } \\ \text { Branch }\end{array} & \begin{array}{l}\text { Direction du } \\ \text { Patrimoine de l'édition }\end{array} \\ \begin{array}{l}\text { 395 Wellington Street } \\ \text { Ottawa ON K1A 0N4 } \\ \text { Canada }\end{array} & \begin{array}{l}\text { 395, rue Wellington } \\ \text { Ottawa ON K1A 0N4 } \\ \text { Canada }\end{array}\end{array}$

Your file Votre référence

ISBN: 978-0-494-44122-0

Our file Notre référence

ISBN: 978-0-494-44122-0

NOTICE:

The author has granted a nonexclusive license allowing Library and Archives Canada to reproduce, publish, archive, preserve, conserve, communicate to the public by telecommunication or on the Internet, loan, distribute and sell theses worldwide, for commercial or noncommercial purposes, in microform, paper, electronic and/or any other formats.

The author retains copyright ownership and moral rights in this thesis. Neither the thesis nor substantial extracts from it may be printed or otherwise reproduced without the author's permission.
AVIS:

L'auteur a accordé une licence non exclusive permettant à la Bibliothèque et Archives Canada de reproduire, publier, archiver, sauvegarder, conserver, transmettre au public par télécommunication ou par l'Internet, prêter, distribuer et vendre des thèses partout dans le monde, à des fins commerciales ou autres, sur support microforme, papier, électronique et/ou autres formats.

L'auteur conserve la propriété du droit d'auteur et des droits moraux qui protège cette thèse. $\mathrm{Ni}$ la thèse ni des extraits substantiels de celle-ci ne doivent être imprimés ou autrement reproduits sans son autorisation.
In compliance with the Canadian Privacy Act some supporting forms may have been removed from this thesis.

While these forms may be included in the document page count, their removal does not represent any loss of content from the thesis.
Conformément à la loi canadienne sur la protection de la vie privée, quelques formulaires secondaires ont été enlevés de cette thèse.

Bien que ces formulaires aient inclus dans la pagination, il n'y aura aucun contenu manquant.

\section{Canada}


Large Deviations for Re-sequencing Buffer Size

\author{
Yanfei Gao
}

2008 


\begin{abstract}
Multi-paths transmission technique is widely used in both advanced wired and wireless network systems and modern high speed digital data transmissions. Multipath routing can potentially help balance the traffic load and reduce congestion levels in the network by sending data packets along different paths, which results in lower delay and higher performance. On the other hand, reliable transportation protocols such as TCP/IP require packets to be delivered to the receiver in the order they are transmitted at the sender. The queue size, which is one of the research interests for the multi-path problem. In this thesis, we derived the large-deviation type of the re-sequencing queue size for the $3-M / M / 1$ queue case and the large-deviation type of the re-sequencing queue size for optimal situation among all Bernoulli routings.
\end{abstract}




\section{Acknowledgements}

I would like to express my gratitude to all those who gave me the possibility to complete this thesis. I am deeply indebted to my supervisor Dr. Yiqiang Zhao from Carleton University, whose help, stimulating suggestions and encouragement helped me in all the time of research for and writing of this thesis.

The Academic Writing Centre of Carleton University supported me look closely at the final version of the thesis for English style and grammar. I want to thank them for all their help, suggestions and corrections.

Especially, I would like to give my special thanks to my husband, Ge Wang, whose patient love, wisdom and encouragement enabled me to complete this work. 


\section{Contents}

$\begin{array}{ll}\text { Abstract } & \text { ii }\end{array}$

Acknowledgements iii

1 Introduction $\quad 1$

1.1 Motivation ......................... 1

1.2 Contribution.......................... 4

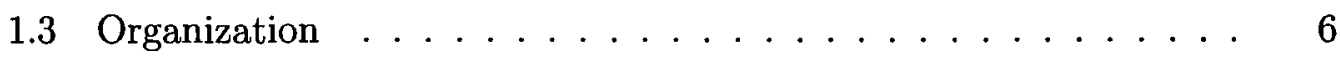

2 Background 7

2.1 General Re-sequence Queue Model . . . . . . . . . . . . . 7

2.1.1 Re-sequencing Caused by Multiple Channels/Paths . . . . 8

2.1.2 Re-sequencing Caused by Retransmissions . . . . . . . . 9 9

2.1.3 Characteristics of Re-sequencing Systems . . . . . . . 10

2.2 Queueing Theory ... . . . . . . . . . . . . 10

2.2 .1 Poisson Process . . . . . . . . . . . . . . . 11

2.2 .2 Queueing Models .................. 13

2.3 Large Deviation . . . . . . . . . . . . . . . . . . . . . 14 
$\begin{array}{lll}3 & 3-M / M / 1 \text { Case } & 16\end{array}$

3.1 Model Description . . . . . . . . . . . . . . . 16

3.1 .1 Setup ............................ 18

3.1.2 The Relationship between $\mathbb{P}\left\{q^{r}(t) \geq n\right\}$ and $M_{i j}(t, s) \ldots 20$

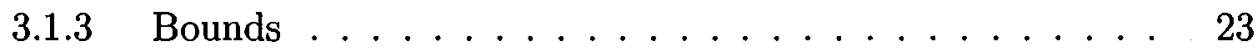

3.2 Computation of $\mathbb{P}\{M(T) \geq n\} \quad \ldots \ldots 28$

3.2.1 Case 1: $\mu_{1}-\lambda_{1}+\mu_{2}-\lambda_{2} \geq \mu_{3}-\lambda_{3} \ldots \ldots 29$

3.2.2 Case 2: $\mu_{1}-\lambda_{1}+\mu_{2}-\lambda_{2}<\mu_{3}-\lambda_{3} \ldots \ldots 39$

3.2.3 Large Deviation Results for $M(T) \ldots \ldots$. . . . . . . . . 49

3.3 Conclusion ..................... 50

$4 m-M / M / 1$ Case $\quad 57$

4.1 Model Description . . . . . . . . . . . . . 57

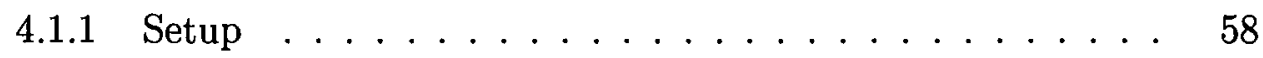

4.1.2 The Relationship between $\mathbb{P}\left\{q^{r}(t) \geq n\right\}$ and $M_{(i)}(t, s) \quad \ldots \quad 59$

4.1 .3 Bounds .................... 60

4.2 Computation of $\mathbb{P}\{\hat{M}(\hat{T}) \geq n\} \quad \ldots \ldots 65$

4.2.1 Case 1: $\sum_{i=1}^{m-1}\left(\mu_{i}-\lambda_{i}\right) \geq \mu_{m}-\lambda_{m} \ldots \ldots 6$

4.2.2 Case $2: \sum_{i=1}^{m-1}\left(\mu_{i}-\lambda_{i}\right)<\mu_{m}-\lambda_{m} \ldots \ldots 69$

4.2 .3 Large Deviation Results for $\hat{M}(\hat{T}) \ldots \ldots 74$

4.3 Conclusion . . . . . . . . . . . . . . . . . 75 


\section{List of Figures}

2.1 Re-sequencing Network Model Followed by a $G / G / 1$ Queue . . . 8

2.2 The Network Model . . . . . . . . . . . . . . . . . . 9 9

$3.13-M / M / 1$ Network and Re-sequencing Model . . . . . . . . 17

$4.1 m-M / M / 1$ Network and Re-sequencing Model . . . . . . . . 58 


\section{Chapter 1}

\section{Introduction}

\subsection{Motivation}

With the increase in transmission speeds, available bandwidth required is also growing steadily. Multi-path transmission technique is widely used in both advanced wired and wireless network systems and modern high speed digital data transmissions. Multi-path routing can potentially help balance the traffic load and reduce congestion levels in the network by sending data packets along different paths, which results in lower delay and higher performance. Transmission over multiple paths also increases the ability of the network to adapt to link failures.

On the other hand, reliable transportation protocols such as TCP/IP require packets to be delivered to the receiver in the order they are transmitted at the sender. Moreover, in many distributed applications, such as voice/data transmissions, remote computations and database manipulations, information integrity 
requires data exchanges between different nodes of a system to be performed in a specific order. However, due to multi-path transmissions, which may have different speeds (available bandwidth), packets may arrive at the destination out of order. Therefore, out-of-order packets must be re-ordered in the transport layer before reaching receiver applications. Thus, re-sequencing becomes an important issue associated with communications through multiple paths.

The re-sequencing problem in queueing networks has some history, as networks of queues in parallel are a natural way to model problems of resource and traffic allocation. Many optimization problems based on them have been studied, a queueing system consisting of $K$ identical single-server queues with infinite buffer size in parallel was considered by Cheng and Chao (1990) in [3]. This paper assumed that each arrival enters one of the $K$ queues with probabilities $p_{i}(i=1,2, \cdots, k)$, and they believed that the expected waiting time and the expected response time of the $n^{\text {th }}$ customer are minimized when $p_{i}=\frac{1}{k}$ for all i. Gün, Jean-Marie, Makowski and Tedijanto[5] and Shanthikumar [11] derived various convexity and monotonicity properties of performance measures in parallel $G / G / 1$ queues with Bernoulli routing and also claimed that the equalikely assignment is optimal among all Bernoulli routings. In view of recent research, Jean-Marie and Gün (1993) [1] considered $k$ parallel $M / G / 1$ queues in the disordering network for which the distributions of re-sequencing and end-to-end delays were derived. Nitin Gogate and Panwar (1994)[10] show that the optimal splitting probability may be heavily dependent on the difference in the fixed delays on the two paths for $2-M / M / 1$ queues. 
Another example of multi-path transmission application is Point to Point (PPP) Multilink Protocol (MP) which was originally introduced in RFC 1717 and updated in RFC 1990 (http://www.ietf.org/rfc/rfc1990.txt). Basically, MP divides the PPP frame into fragments that are evenly sent out over multiple physical links. In order to establish communications over a point-to-point link, each end of the PPP link must first send LCP (Link Control Protocol, RFC 1661, http://www.ietf.org/rfc/rfc1661.txt) packets to configure the data link during the link establishment phase. After the link has been established, PPP provides an authentication phase in which the authentication protocols can be used to determine identifiers associated with each system connected by the link. The goal of multilink operation is to coordinate multiple independent links between a fixed pair of systems, providing a virtual link with greater bandwidth than any of the constituent members. The bundled links can be different physical links, such as pairing dial-up asynchronous links with leased synchronous links. The packets transmitted using the multilink procedure are encapsulated according to the rules for PPP where no link quality monitoring is required. LCP negotiations are not permitted on the bundle itself. An implementation must not transmit LCP configure such as -Request, -Reject, -Ack, -Nak, Terminate-Request or -Ack packets via the multilink procedure. Also, there are no requirements that the segments are of equal sizes, or that packets must be broken up at all. A possible strategy for contending with member links of differing transmission rates would be to divide the packets into segments proportional to the transmission rates. Another strategy might be to divide them into many equal fragments and distribute multiple fragments per link, the numbers being proportional to the relative speeds of the 
links.

More recently, Han and Makowski (2006) [6] pointed out that the asymptotic in the heavy-tailed cases is radically different from the light-tailed asymptotic under multi-path routing. Lelarge (2008) [9] derived the exact asymptotic of the packet re-sequencing delay and the re-sequencing queue size for the $2-\cdot / G / 1$ under the heavy-tail assumption.

The buffer size, which is one of the research interests for the multi-path problem, was studied by Xia and Tse (2004) in an earlier paper [13], where the disordering is caused by the 2-path routings and the large deviation result for the re-sequencing queue size was derived. In this thesis, we extend the research to three or more transmission paths and study the behavior of the re-sequencing queue size due to different path circumstances. For simplicity, in this thesis we assume that there are no packet transmission errors across each of these paths, that is, there are no feedback and no retransmission. We concentrate on the resequencing queue size analysis due to multi-paths transmission, as well as different service rates.

\subsection{Contribution}

In multi-path data communication systems, it is often assumed that packets are generated according to a Poisson process, but unlike packet transmission over a 
single channel, packets are transmitted over multiple routes with certain probabilities. In this thesis, a network model with three parallel queues, each of which having an infinite capacity buffer is considered. Packets arrive according to a Poisson process and are allocated to the queues by a Bernoulli switch. Service times at different queues are assumed to be independent but need not be identically distributed. Each packet is subject to re-sequencing so that after completing service, it moves to a re-sequencing queue and eventually awaits the service completion of the jobs that have arrived into the system before it. The model is introduced precisely in Chapter 3. The main contributions of this thesis are stated as follows:

The large-deviation type of the re-sequencing queue size for the $3-$ $M / M / 1$ queue case

The paper [13] only discusses $2-M / M / 1$ case, but in fact more than 2 links may be used to increase the transmission speed. Main result in Chapter 3 allows us to get large-deviation type of the re-sequencing queue size for three parallel independent $M / M / 1$ queues with different arrivals and services. Limit theorems are used to obtain both a lower and an upper bounds of the event which converge to the same value. Like most large deviation problems, usually one bound is easy to find; while the other needs more efforts. The lower bound of situation 3.2.1 and upper bound in 3.2.2 are more challenging to address. Thus in the analysis we use the fact that the departure of $2-M / M / 1$ with arrival rate $\lambda_{1}, \lambda_{2}$ has the same distribution as the $M / M / 1$ queue with the arrival rate $\lambda_{1}+\lambda_{2}$. Then, it becomes feasible to find the bounds through analyzing one queue instead of a 
system consisting of more than one queue. In Chapter 4, we also use the similar method to obtain the main result for the $m-M / M / 1$ case.

The large-deviation type of the re-sequencing queue size for optimal situation among all Bernoulli routings

Since [3], [5] and [11] has shown that the optimal situation among all Bernoulli routings for $k$ identical parallel single-sever queues is when all $p_{i}$ are equal. In Chapter 4 , we prove if the disordering network has $m$ independent identical $M / M / 1$ queues having arrival rate $\lambda$ and service rate $\mu$, then the large deviation type of re-sequencing queue size always has the form of $\log \frac{(m-1) \lambda}{(m-2) \lambda+\mu}$. Furthermore, it is observed that as the increase the number of queues in disordering network, the large-deviation type of the re-sequencing queue size is also increasing.

\subsection{Organization}

The thesis is organized as follows: Chapter 2 gives the general re-sequencing queue models and some preliminary results of the queueing theory, which are necessary to this thesis; we also introduce principles of large deviations. In Chapter 3 , we describe the re-sequencing model of $3-M / M / 1$ case and give one of the main results: Theorem 3.2. In Chapter 4, we try to find the general expression of the lager deviation type of queue size, $\lim _{n \rightarrow \infty} \frac{1}{n} \log \mathbb{P}\left\{q^{r}(t) \geq n\right\}$ for $m-M / M / 1$ case, where $m \geq 2$. For simplicity, we assume all these $m M / M / 1$ queues are independent and identical. 


\section{Chapter 2}

\section{Background}

This chapter provides the background information relevant to the research on re-sequencing queue buffer size reported in this thesis, and it is organized as follows. Section 2.1 defines a general network model including a re-sequence agent and briefly describes the packet re-sequencing problem in network transmission. Section 2.2 is an overview of some well-known results in queue theory which will be used in later chapters. Section 2.3 introduces some fundamental principles of large deviations, which will be identified for the re-sequencing queue size of the models studied.

\subsection{General Re-sequence Queue Model}

Internet Transmission Protocols such as TCP require packets to be accepted by the receiver in the order they are transmitted at the sender, that is, the receiving application can only recognize ordered packets. However, packets that arrive at the receiving host may be disordered during transmission for several reasons. First, 
for efficiency and reliability reasons, logical channels connecting adjacent nodes of a virtual circuit may consist of multiple physical links, which means packets are usually transmitted over multiple parallel channels/paths given the fact that every packet contains the destination address. Due to different channel/path transmission speeds, packet traveling lengths, packet sizes, channel conditions, etc. Packets may arrive at the destination node in an order different from that in which they were originally generated at the source node. Second, a packet may be dropped by the network when the network is congested or by the receiver if the packet is corrupted. For reliable communication, the sender must retransmit the dropped packets, possibly resulting in out-of-order reception. In order to achieve sequenced delivery of the arrived packets to the application, packets need to be properly reordered with the help of a re-sequencing buffer.

\subsubsection{Re-sequencing Caused by Multiple Channels/Paths}

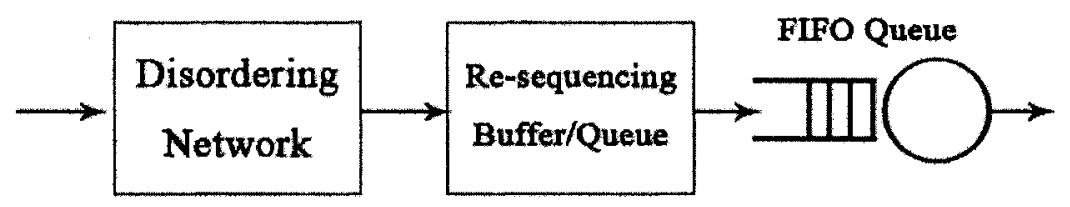

Figure 2.1: Re-sequencing Network Model Followed by a $G / G / 1$ Queue

Studies on packet disordering caused by multiple paths typically analyze an open queuing network, with no feedback and no retransmission. A general re-sequencing 
network model is shown in Figure 2.1. This model consists of three parts: a disordering network, a re-sequencing buffer (re-sequencing queue), and a queue given that First-In-First-Out (FIFO) delivery is required, a FIFO queue always follows the re-sequencing buffer.

Packets arriving at the DN are numbered in a numerical sequence. For example, the sequence $P_{1}, P_{2}, P_{3}, \cdots$, represents a sequence of arriving packets, where $P_{n}$ is the $n^{\text {th }}$ packet arriving at the DN. However, packet $P_{n}$ may leave the DN before packet $P_{k}$ with $k<n$, since they pass the DN by different paths. Usually, the disordering network is also modeled as a queuing system, whose type typically distinguishes in different studies. The problem analyzed in this thesis is a special case of Figure 2.1.

\subsubsection{Re-sequencing Caused by Retransmissions}

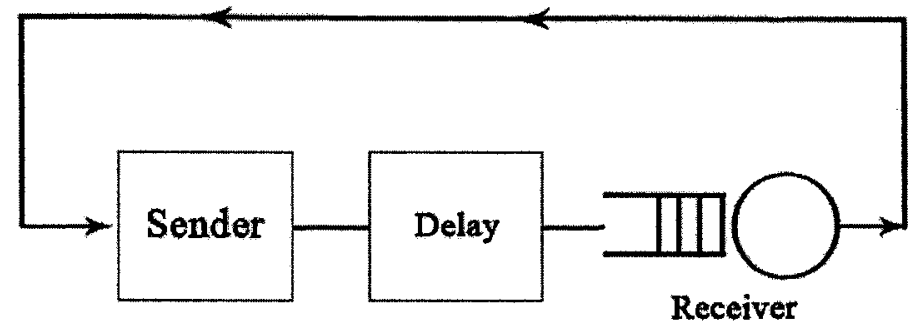

Figure 2.2: The Network Model

Another common situation of out-of-order problem is caused by retransmission. An automatic repeat-request (ARQ) model is often used in such circumstances 
shown in Figure 2.2. ARQ is typically considered as a link layer protocol running between a sender-receiver pair over a link with constant propagation delay. When there is noise in the communication channel from the sender to the receiver, the sender needs to retransmit the corrupted packets based on the feedback information from the receiver. Several papers deal with the re-sequencing problems at the receiver caused by packet retransmission, such as [14] and [15]. This type of re-sequence problem will not be considered in this thesis.

\subsubsection{Characteristics of Re-sequencing Systems}

The re-sequencing delay is an important characteristic for evaluating the performance of the model, which is defined as the waiting time of a packet in the resequencing buffer. Various models for analyzing re-sequencing problems are studied and exact expressions for the re-sequencing delay distributions are obtained. Another characteristic is the re-sequencing buffer size, which is determined by the distribution of the number of packets in the re-sequence buffer.

In this thesis, we focus on the buffer size problem of the re-sequencing system caused by multiple paths.

\subsection{Queueing Theory}

In this section, we will review some basic conclusions in queueing theory especially the results of the single-server exponential queueing system $(M / M / 1)$. 


\subsubsection{Poisson Process}

Definition 2.1 The counting process $\{N(t), t \geq 0\}$ is said to be a Poisson process having rate $\lambda, \lambda \geq 0$, if

- $N(0)=0$;

- The process has independent increments;

- The number of events in any interval of length $t$ is Poisson distributed with mean $\lambda t$. That is, for all $s, t \geq 0$

$$
\mathbb{P}\{N(t+s)-N(s)=n\}=e^{-\lambda t} \frac{(\lambda t)^{n}}{n !} \quad n=0,1, \cdots
$$

A counting process is said to have stationary increments if the distribution of the number of events that occur in any interval of time just depends on the length of the time interval. From the definition above, it is easy to know that Poisson process has stationary increments.

An equivalent definition of Poisson process is stated as follows (Proof can be found in [12]).

Definition 2.2 The counting process $\{N(t), t \geq 0\}$ is said to be a Poisson process having rate $\lambda, \lambda \geq 0$, if

- $N(0)=0$;

- The process has stationary and independent increments;

- $\mathbb{P}\{N(h)=1\}=\lambda h+o(h)$;

- $\mathbb{P}\{N(h) \geq 2\}=o(h)$, 
where $o(h)$ is a function such that $\lim _{h \rightarrow 0} \frac{o(h)}{h}=0$.

Consider a Poisson process $\{N(t), t \geq 0\}$ with rate $\lambda$, let $T_{n}$ denote the elapsed time between the $(n-1)^{s t}$ and the $n^{\text {th }}$ events. The sequence $\left\{T_{n}, n=1,2, \cdots\right\}$ is called the sequence of interarrival times.

Proposition 2.1 $T_{n}, n=1,2, \cdots$, are independent identically distributed exponential random variables having rate $\lambda$.

For the Poisson process $\{N(t), t \geq 0\}$, suppose that each time an event is classified as either a type I event with probability $p$ or a type II event with probability $1-p$, independently of all other events. Let $N_{1}(t)$ and $N_{2}(t)$ denote the number of type I and type II events occurring in time interval $[0, t]$, where $N(t)=N_{1}(t)+N_{2}(t)$.

Proposition 2.2 (Poisson Split Property) $\left\{N_{1}(t), t \geq 0\right\}$ and $\left\{N_{2}(t), t \geq 0\right\}$ are both Poisson processes with rate $\lambda p$ and $\lambda(1-p)$, respectively. Furthermore, the two process are independent.

Proof. It is easy to verify that $\left\{N_{1}(t), t \geq 0\right\}$ and $\left\{N_{2}(t), t \geq 0\right\}$ are Poisson processes with rate $\lambda p$ and $(1-\lambda) p$ by definition 2.2 . Detail can be found in [12] (Page 296).

Because a type I event in the interval $(t, t+h)$ is independent of any type I event occurring in an interval that does not overlap $(t, t+h)$, and furthermore, it is independent of any type II event, which indicates that the two Poisson process are independent. 


\subsubsection{Queueing Models}

Suppose that customers arrive at a single-server service station with a Poisson process having rate $\lambda$, that is, the time between successive arrivals are independent exponential random variables with rate $\lambda$. Each customer, upon arrival, goes directly into the service if the server is idle; otherwise if there is another customer being served at this moment, the new-coming customer will join the queue. When the server finishes serving a customer, the customer leaves the system and the next customer (if there is any) in the queue will be served immediately. The successive service times are assumed to be independent exponential random variables having rate $\mu$.

The preceding process is called the $M / M / 1$ queue, where the two $M \mathrm{~s}$ refer to the fact that both the inter-arrival and the service distributions are exponential, and the 1 to the fact that there is a single server. Sometimes, we denote a queue as $M / M / 1 / N$ or $M / M / 1 / \infty$, where the last part is the capacity of this queue, that is, the total number of customers that could be in the system at the same time. Moreover, if there is more than one server in the system we can use $M / M / n$.

Furthermore, in $G / G / n / N$, the times between successive arrivals and services have arbitrary distribution $G$, and $n, N$ are the same as we denoted before. Similarly, there are many different kinds of queues, such as $M / G / 1, G / M / 1$, $G / G / 2, \ldots$. In this thesis, we mainly discuss the $M / M / 1$ queue.

Proposition 2.3 (Stability Condition for $M / M / 1$ Queue) If $\rho\left(=\frac{\lambda}{\mu}\right)<1$ then 
the queue is stable and has a unique stationary probability distribution. If $\rho \geq 1$ then the queue size grows to infinity.

Let $P_{i}$ be the proportion of time in state $i$ and $q_{i j}$ be the rate when in state $i$ that the process goes to $j$.

Definition 2.3 The continuous Markov chain is time reversible, in the sense that a process reversed in time has the same probabilistic structure as the original process, if $P_{i} q_{i j}=P_{j} q_{j i}$, for all $i, j$.

Proposition 2.4 An ergodic birth and death process is time reversible.

Proposition 2.5 $M / M / 1$ queue is an ergodic queueing process if $\lambda<\mu$.

Corollary 2.1 Consider an $M / M / 1$ queue with arrival rate $\lambda$, and service rate $\mu(\lambda<\mu)$, then the output process of customers departing is, after the process has been in operation for a long time, a Poisson process with rate $\lambda$.

\subsection{Large Deviation}

Large deviation theory is a part of probability theory that deals with the description of events where a sum of random variables deviates beyond what is described by the central limit theorem, or more generally, a precise calculation of the probabilities of such events turns out to be crucial for the study of integrals of exponential functional of sums of random variables. 
In this thesis, the large deviation type of queue size we discussed is stated as $\lim _{n \rightarrow \infty} \frac{1}{n} \log \mathbb{P}\left\{q^{r}(t) \geq n\right\}$. Moreover, when we discuss the large deviation problem, we need to make some restrictions such that:

$$
X_{i} \in \Gamma=1,2, \cdots, v \subset \mathbb{N}
$$

$X_{1}, X_{2}, \cdots$ is Markov with transition matrix $P=\left(P_{s t}\right)_{s, t \in \Gamma}, P_{s t}>0$, for any $s, t \in \Gamma$.

The stationary probability distribution $\pi=\pi_{s}$ of the Markov chain is unique and satisfies

$$
\pi_{s}>0, \quad \forall s \in \Gamma
$$

We pick as initial distribution

$$
\mathbb{P}\left(X_{1}=s\right)=\pi_{s}, \quad \forall s \in \Gamma
$$

so the Markov chain is stationary. For continuous Markov chain $X(t), t \geq 0$, we use $\mathbb{P}(X(0)=j)=P_{j}$ as our initial distribution.

Thus, when we compute $\mathbb{P}\{M(T) \geq n\}$ in Section 3.2 and $\mathbb{P}\{\hat{M}(\hat{T}) \geq n\}$ in Section 4.2 , we assume that all $M / M / 1$ queues are already stationary. More details can be found in reference [4] and [2]. 


\section{Chapter 3}

\section{$3-M / M / 1$ Case}

In this chapter we will describe the disordering network and the re-sequencing model in detail, and state one of the main results in this thesis.

\subsection{Model Description}

Consider a queue, where sequentially numbered customers (or packets, jobs) arrive at the disordering network (DN) according to a Poisson process with rate $\lambda$. As Figure 3.1 shows, there are three parallel single-server queues in the DN. Each server serves one customer at a time with an exponential service time with rate $\mu_{i}, i=1,2,3$ and these three servers are independent. Upon arrival, the customer independently enters queue 1 , queue 2 or queue 3 with probabilities $p_{1}>0, p_{2}>0$ and $p_{3}>0$ respectively, where $p_{1}+p_{2}+p_{3}=1$. In each queue, an arriving customer immediately starts service if the server is idle; otherwise, the customer waits in a line (that is, he/she joins the queue). We assume that there is infinite waiting space at each server. By Poisson Split Property, the arrival processes to the three 


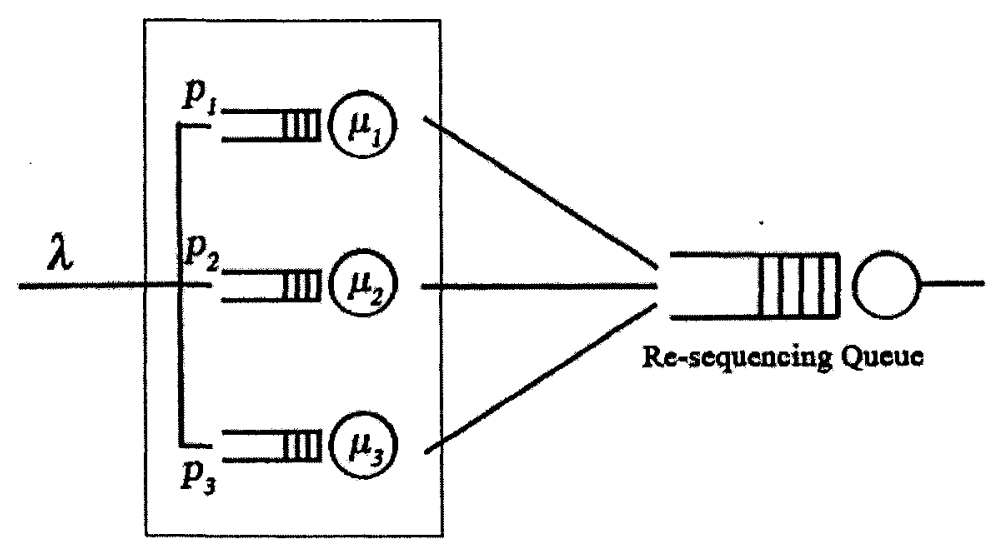

Disordering Network

Figure 3.1: $3-M / M / 1$ Network and Re-sequencing Model

queues are independent and Poisson distributed with rate $\lambda_{1}=p_{1} \lambda, \lambda_{2}=p_{2} \lambda$ and $\lambda_{3}=p_{3} \lambda$, respectively. We assume $\lambda_{i}<\mu_{i}$, so that each queue is stable. Hence, in the DN there are three independent $M / M / 1$ queues with parameter $\lambda_{i}$ and $\mu_{i}$, respectively.

When the server completes serving a customer, the customer leaves the DN system immediately. As there are three parallel queues and each customer enters one of the three queues randomly with specified probabilities, such that different customers may have different sojourn times through the queue. As a result, the customer with a large label could finish the service earlier than one with a smaller label. Therefore, we need to re-sequence customers at the re-sequencing queue (RSQ). Here, we assume that the capacity of RSQ is infinite. If the customers are 
properly ordered in the RSQ, they are immediately transmitted, that is, the $j^{\text {th }}$ customer will leave the RSQ if all customers $i$ have arrived in the RSQ system, where $i<j$. Let $q^{r}$ be the stationary size of the RSQ. We are interested in the large deviation type of result about $q^{r}$.

\subsubsection{Setup}

Let $V(t)$ be the event $=\{\mathrm{DN}$ is empty at time $t\}$. If $V(t)$ does not occur, then there is at least one customer in the DN. Hence, in the whole DN we can always find a customer that has the smallest label (the oldest customer) at time $t$. Compared with other customers, this customer has spent the longest time in DN. We assume that the oldest customer in the DN at time $t$ is $C_{*}(t)$. Let $W_{*}(t)$ be the time the oldest costumer $C_{*}(t)$ has spent in the $\mathrm{DN}$, and let $I_{*}(t)$ be the queue in the DN which $C_{*}(t)$ goes though.

For $n \geq 0$, denote $E(t, s, n)=$ at least $n$ customers arrived at the DN on the interval $(t-s, t]$, among which at least $n$ have left the DN by time $t\}$.

Let $q^{r}(t)$ be the size of the RSQ at time $t$, and let $q_{i}(t)$ be the size of queue $i$ at time $t$, where $i=1,2,3$. At time $t$, the RSQ size is greater or equal to $n$, where $n>0$, means that the DN system is not empty and the $C_{*}(t)$ is being served in $I_{*}(t)$. Therefore, in this situation, all the customers in the RSQ are exactly those who arrived at DN later than $C_{*}(t)$, but who have left the DN by time $t$. Hence, 
for $n>0$,

$$
\mathbb{P}\left\{q^{r}(t) \geq n\right\}=\mathbb{P}\left\{V^{c}(t) \bigcap E\left(t, W_{*}(t), n\right)\right\}
$$

Now, we extend the definition of $W_{*}(t)$ as follows: If $V(t)$ occurs, let $W_{*}(t)=0$. Then, for $n>0$,

$$
\begin{aligned}
\mathbb{P}\left\{V(t) \bigcap E\left(t, W_{*}(t), n\right)\right\} & =\mathbb{P}\left\{E\left(t, W_{*}(t), n\right) \mid V(t)\right\} \mathbb{P}\{V(t)\} \\
& =\mathbb{P}\{E(t, 0, n) \mid V(t)\} \mathbb{P}\{V(t)\} \\
& =0 \cdot \mathbb{P}\{V(t)\}=0
\end{aligned}
$$

As a result, equation(3.1) can be written as

$$
\begin{aligned}
\mathbb{P}\left\{q^{r}(t) \geq n\right\} & =\mathbb{P}\left\{V^{c}(t) \bigcap E\left(t, W_{*}(t), n\right)\right\}+\mathbb{P}\left\{V(t) \bigcap E\left(t, W_{*}(t), n\right)\right\} \\
& =\mathbb{P}\left\{E\left(t, W_{*}(t), n\right)\right\}
\end{aligned}
$$

The expression is also valid for $n=0$,

$$
\begin{aligned}
\mathbb{P}\left\{V^{c}(t) \bigcap E\left(t, W_{*}(t), 0\right)\right\} & =\mathbb{P}\left\{E\left(t, W_{*}(t), 0\right) \mid V^{c}(t)\right\} \mathbb{P}\left\{V^{c}(t)\right\} \\
& =1 \cdot \mathbb{P}\left\{V^{c}(t)\right\}=\mathbb{P}\left\{V^{c}(t)\right\}
\end{aligned}
$$

In the above equation, $\mathbb{P}\left\{E\left(t, W_{*}(t), 0\right) \mid V^{c}(t)\right\}$ is 1 , because by the definition of $E\left(t, W_{*}(t), n\right)$, the probability of event \{at least 0 customers arrived at the DN on the interval $(t-s, t]$, among which at least 0 have left the DN by time t $\}$ equals 1 . 
CHAPTER 3. $3-M / M / 1 C A S E$

Similarly, we can get

$$
\mathbb{P}\left\{V(t) \bigcap E\left(t, W_{*}(t), 0\right)\right\}=\mathbb{P}\{V(t)\}
$$

Add (3.4) and (3.5), we have

$$
\mathbb{P}\left\{E\left(t, W_{*}(t), 0\right)\right\}=1
$$

On the other hand, when $n=0$,

$$
\mathbb{P}\left\{q^{r}(t) \geq 0\right\}=1
$$

Hence,

$$
\mathbb{P}\left\{q^{r}(t) \geq 0\right\}=\mathbb{P}\left\{E\left(t, W_{*}(t), 0\right)\right\}
$$

Combining (3.3) and (3.7), we can write, for any $n \geq 0$,

$$
\mathbb{P}\left\{q^{r}(t) \geq n\right\}=\mathbb{P}\left\{E\left(t, W_{*}(t), n\right)\right\}
$$

\subsubsection{The Relationship between $\mathbb{P}\left\{q^{r}(t) \geq n\right\}$ and $M_{i j}(t, s)$}

Let $M_{i j}(t, s)$ be the total number of customers who arrived at the queue $i$ or queue $j$ during the time interval $(t-s, t]$ and departed by time $t$, where $i \neq j$ and $i, j=1,2,3$. 
Note that in the DN system, the customers are served based on first-comefirst-served rule in each of the three queues. Therefore, at time $t$, if the DN is non-empty, the oldest customer $C_{*}(t)$ must be in service at the queue $I_{*}(t)$. Obviously, all customers in the RSQ must arrive at the DN after the oldest customer and go through the other two queues. For example, suppose that the oldest customer is being served at queue 1 , then $q^{r}$ equals to the total number of customers who went through queue 2 and queue 3 during the time that the oldest customer spent in queue 1.

At time $t$, if queue $i$ is not empty, $i=1,2,3$, let $W_{i}(t)$ be the duration for which the customer in service at queue $i$ has stayed in the queue. If the queue is empty, define $W_{i}(t)=0$. By using a simple reversibility argument, $W_{i}(t)$ has the same distribution as the waiting time in queue $i$ (not including the service time) by an arbitrary customer. Hence, the distribution and density functions are (page 213 in [8]), for $x \geq 0$,

$$
\begin{aligned}
& F_{W_{i}}(x)=\mathbb{P}\left\{W_{i}(t) \leq x\right\}=1-\rho_{i} \exp \left\{-\left(\mu_{i}-\lambda_{i}\right) x\right\} \\
& f_{W_{i}}(x)=\left(1-\rho_{i}\right) \delta(x)+\lambda_{i}\left(1-\rho_{i}\right) \exp \left\{-\left(\mu_{i}-\lambda_{i}\right) x\right\}
\end{aligned}
$$

where $\rho_{i}=\lambda_{i} / \mu_{i}$, and $\delta(x)$ is the Dirac delta function, representing the point probability mass at $x=0$. 
Note that, for $n>0$,

$$
\begin{aligned}
& \mathbb{P}\left\{M_{12}\left(t, W_{*}(t)\right) \geq n \mid W_{1}(t)=W_{2}(t)=W_{3}(t)=0\right\} \\
= & \mathbb{P}\left\{M_{12}(t, 0) \geq n \mid W_{1}(t)=W_{2}(t)=W_{3}(t)=0\right\} \\
= & 0
\end{aligned}
$$

The last probability equals 0 , because when $n>0$ and given $W_{1}(t)=W_{2}(t)=$ $W_{3}(t)=0$, the probability of event $\{$ the total number of customers who arrived at queue 1 or queue 2 during the time interval $(t-0, t]$ is greater than $n\}$ is equals 0 .

Also, since $W_{i}(t)$ is a continuous random variable in $(0, \infty)$, we have

$$
\mathbb{P}\left\{W_{1}(t)=W_{2}(t)=W_{3}(t) \neq 0\right\}=\mathbb{P}\left\{W_{i}(t)=W_{j}(t) \neq 0\right\}=0
$$

where, $i, j \in\{1,2,3\}$ and $i \neq j$.

Therefore, it is easy to check the following equation,

$$
\begin{aligned}
& \mathbb{P}\left\{M_{i j}\left(t, W_{*}(t)\right) \geq n \mid W_{i}(t)=W_{k}(t), W_{j}(t) \geq W_{k}(t)\right\} \\
& \mathbb{P}\left\{W_{i}(t)=W_{k}(t), W_{j}(t) \geq W_{k}(t)\right\} \\
= & 0
\end{aligned}
$$

where $\{i, j, k\} \in\{1,2,3\}$ and they are not equal.

From the definition of $W_{i}(t)$ and the analysis above, the relationship between $\mathbb{P}\left\{q^{r}(t) \geq n\right\}$ and $M_{i j}(t, s)$ can be explained as follows: 
At time $t$, if $W_{1}(t)>W_{2}(t)$ and $W_{1}(t)>W_{3}(t)$, the oldest customer, $C_{*}(t)$, in the DN must be in service at queue 1. Hence $W_{1}(t)=W_{*}(t)$. All customers who came to the DN after $C_{*}(t)$ and also left the DN by time $t$ must have been routed to the RSQ from queue 2 or queue 3 , which means that at this situation, $\mathbb{P}\left\{q^{r}(t) \geq n\right\}$ equals to $\mathbb{P}\left\{M_{23}\left(t, W_{*}(t)\right) \geq n\right\}$. Similarly, we can discuss the other two cases, $\left\{W_{2}(t)>W_{1}(t)\right.$ and $\left.W_{2}(t)>W_{3}(t)\right\}$ and $\left\{W_{3}(t)>W_{1}(t)\right.$ and $\left.W_{3}(t)>W_{2}(t)\right\}$

Therefore, for $n>0$,

$$
\begin{aligned}
& \mathbb{P}\left\{q^{r}(t) \geq n\right\} \\
= & \mathbb{P}\left\{E\left(t, W_{*}(t), n\right)\right\} \\
= & \mathbb{P}\left\{M_{23}\left(t, W_{*}(t)\right) \geq n \mid W_{1}(t)>W_{2}(t), W_{1}(t)>W_{3}(t)\right\} \\
& \cdot \mathbb{P}\left\{W_{1}(t)>W_{2}(t), W_{1}(t)>W_{3}(t)\right\} \\
& +\mathbb{P}\left\{M_{13}\left(t, W_{*}(t)\right) \geq n \mid W_{2}(t)>W_{1}(t), W_{2}(t)>W_{3}(t)\right\} \\
& \cdot \mathbb{P}\left\{W_{2}(t)>W_{1}(t), W_{2}(t)>W_{3}(t)\right\} \\
& +\mathbb{P}\left\{M_{12}\left(t, W_{*}(t)\right) \geq n \mid W_{3}(t)>W_{1}(t), W_{3}(t)>W_{2}(t)\right\} \\
& \cdot \mathbb{P}\left\{W_{3}(t)>W_{1}(t), W_{3}(t)>W_{2}(t)\right\}
\end{aligned}
$$

\subsubsection{Bounds}

From the discussion in Section 3.1.2, we note that the $\mathbb{P}\left\{q^{r}(t) \geq n\right\}$ consists of three symmetric parts: $\mathbb{P}\left\{M_{23}\left(t, W_{*}(t)\right) \geq n \mid W_{1}(t)>W_{2}(t), W_{1}(t)>W_{3}(t)\right\}$, $\mathbb{P}\left\{M_{13}\left(t, W_{*}(t)\right) \geq n \mid W_{2}(t)>W_{1}(t), W_{2}(t)>W_{3}(t)\right\}$ and $\mathbb{P}\left\{M_{12}\left(t, W_{*}(t)\right) \geq\right.$ 
$\left.n \mid W_{3}(t)>W_{1}(t), W_{3}(t)>W_{2}(t)\right\}$. Without losing generality, in this subsection, we just discuss the component $\mathbb{P}\left\{M_{23}\left(t, W_{*}(t)\right) \geq n \mid W_{1}(t)>W_{2}(t), W_{1}(t)>\right.$ $\left.W_{3}(t)\right\}$. Using the same method we can easily obtain the bounds for the other two cases.

For $n>0$, conditioning on $W_{1}(t)$

$$
\begin{aligned}
& \mathbb{P}\left\{M_{23}\left(t, W_{*}(t)\right) \geq n \mid W_{1}(t)>W_{2}(t), W_{1}(t)>W_{3}(t)\right\} \\
= & \int_{0+}^{\infty} \mathbb{P}\left\{M_{23}(t, s) \geq n \mid W_{1}(t)=s, W_{1}(t)>W_{2}(t), W_{1}(t)>W_{3}(t)\right\} \\
& \cdot f_{W_{1} \mid W_{1}>W_{2}, W_{1}>W_{3}}(s) d s \\
= & \int_{0+}^{\infty} \mathbb{P}\left\{M_{23}(t, s) \geq n \mid W_{2}(t)<s, W_{3}(t)<s\right\} \\
& \cdot f_{W_{1} \mid W_{1}>W_{2}, W_{1}>W_{3}}(s) d s
\end{aligned}
$$

In the above, $f_{W_{1} \mid W_{1}>W_{2}, W_{1}>W_{3}}(s)$ denote the conditional density of $W_{1}(t)$ given $\left\{W_{1}(t)>W_{2}(t), W_{1}(t)>W_{3}(t)\right\}$. For the second equality, we used the fact that the three queues are independent. Note that, the integral in this equation is from $0+$ to $\infty$, the conditional probability mass at $s=0$ does not contribute to the probability on the left hand side.

To compute $f_{W_{1} \mid W_{1}>W_{2}, W_{1}>W_{3}}(s)$, we start with the joint probability of $\left\{W_{1}(t)>\right.$ $\left.x, W_{1}(t)>W_{2}(t), W_{1}(t)>W_{3}(t)\right\}$ 
For $x \geq 0$, still by conditioning on $W_{1}(t)$,

$$
\begin{aligned}
& \mathbb{P}\left\{W_{1}(t)>x, W_{1}(t)>W_{2}(t), W_{1}(t)>W_{3}(t)\right\} \\
= & \int_{x+}^{\infty} \mathbb{P}\left\{W_{2}(t)<s, W_{3}(t)<s \mid W_{1}(t)=s\right\} f_{W_{1}}(t) d s \\
= & \int_{x+}^{\infty} \mathbb{P}\left\{W_{2}(t)<s\right\} \mathbb{P}\left\{W_{3}(t)<s\right\} f_{W_{1}}(t) d s \quad \text { (by independence) } \\
= & \int_{x+}^{\infty}\left[1-\rho_{2} e^{-\left(\mu_{2}-\lambda_{2}\right) s}\right]\left[1-\rho_{3} e^{-\left(\mu_{3}-\lambda_{3}\right) s}\right] \lambda_{1}\left(1-\rho_{1}\right) e^{-\left(\mu_{1}-\lambda_{1}\right) s} \\
= & \rho_{1} e^{-\left(\mu_{1}-\lambda_{1}\right) x}-\rho_{1} \rho_{2} \frac{\mu_{1}-\lambda_{1}}{\mu_{1}-\lambda_{1}+\mu_{2}-\lambda_{2}} e^{-\left(\mu_{1}-\lambda_{1}+\mu_{2}-\lambda_{2}\right) x} \\
& -\rho_{1} \rho_{3} \frac{\mu_{1}-\lambda_{1}}{\mu_{1}-\lambda_{1}+\mu_{3}-\lambda_{3}} e^{-\left(\mu_{1}-\lambda_{1}+\mu_{3}-\lambda_{3}\right) x} \\
& +\rho_{1} \rho_{2} \rho_{3} \frac{\mu_{1}-\lambda_{1}}{\mu_{1}-\lambda_{1}+\mu_{2}-\lambda_{2}+\mu_{3}-\lambda_{3}} e^{-\left(\mu_{1}-\lambda_{1}+\mu_{2}-\lambda_{2}+\mu_{3}-\lambda_{3}\right) x}
\end{aligned}
$$

To compute $\mathbb{P}\left\{W_{1}(t)>W_{2}(t), W_{1}(t)>W_{3}(t)\right\}$, we just need to find the marginal distribution of $W_{1}(t)$ in the above equation. So from (3.13), we have

$$
\begin{aligned}
& \mathbb{P}\left\{W_{1}(t)>W_{2}(t), W_{1}(t)>W_{3}(t)\right\} \\
= & \mathbb{P}\left\{W_{1}(t)>0, W_{1}(t)>W_{2}(t), W_{1}(t)>W_{3}(t)\right\} \\
= & \rho_{1}-\rho_{1} \rho_{2} \frac{\mu_{1}-\lambda_{1}}{\mu_{1}-\lambda_{1}+\mu_{2}-\lambda_{2}}-\rho_{1} \rho_{3} \frac{\mu_{1}-\lambda_{1}}{\mu_{1}-\lambda_{1}+\mu_{3}-\lambda_{3}} \\
& +\rho_{1} \rho_{2} \rho_{3} \frac{\mu_{1}-\lambda_{1}}{\mu_{1}-\lambda_{1}+\mu_{2}-\lambda_{2}+\mu_{3}-\lambda_{3}}
\end{aligned}
$$

From (3.13) and (3.14), we get the conditional density, $f_{W_{1} \mid W_{1}>W_{2}, W_{1}>W_{3}}(x)$. 
For $x \geq 0$,

$$
\begin{aligned}
& \bar{F}_{W_{1} \mid W_{1}>W_{2}, W_{1}>W_{3}}(x) \\
= & \frac{\mathbb{P}\left\{W_{1}(t)>x, W_{1}(t)>W_{2}(t), W_{1}(t)>W_{3}(t)\right\}}{\mathbb{P}\left\{W_{1}(t)>W_{2}(t), W_{1}(t)>W_{3}(t)\right\}}
\end{aligned}
$$

Thus,

$$
\begin{aligned}
& f_{W_{1} \mid W_{1}>W_{2}, W_{1}>W_{3}}(x) \\
= & \frac{d}{d x}\left(1-\bar{F}_{W_{1} \mid W_{1}>W_{2}, W_{1}>W_{3}}(x)\right) \\
= & K_{1} e^{-\left(\mu_{1}-\lambda_{1}\right) x}-K_{2} e^{-\left(\mu_{1}-\lambda_{1}+\mu_{2}-\lambda_{2}\right) x}-K_{3} e^{-\left(\mu_{1}-\lambda_{1}+\mu_{3}-\lambda_{3}\right) x} \\
& +K_{4} e^{-\left(\mu_{1}-\lambda_{1}+\mu_{2}-\lambda_{2}+\mu_{3}-\lambda_{3}\right) x}
\end{aligned}
$$

where $K_{1}, K_{2}, K_{3}$ and $K_{4}$ are positive constants, given by,

$$
\begin{aligned}
& K_{1}=\frac{\mu_{1}-\lambda_{1}}{1-\rho_{2} \frac{\mu_{1}-\lambda_{1}}{\mu_{1}-\lambda_{1}+\mu_{2}-\lambda_{2}}-\rho_{3} \frac{\mu_{1}-\lambda_{1}}{\mu_{1}-\lambda_{1}+\mu_{3}-\lambda_{3}}+\rho_{1} \rho_{2} \rho_{3} \frac{\mu_{1}-\lambda_{1}}{\mu_{1}-\lambda_{1}+\mu_{2}-\lambda_{2}+\mu_{3}-\lambda_{3}}} \\
& K_{2}=\frac{\rho_{2}\left(\mu_{1}-\lambda_{1}\right)}{1-\rho_{2} \frac{\mu_{1}-\lambda_{1}}{\mu_{1}-\lambda_{1}+\mu_{2}-\lambda_{2}}-\rho_{3} \frac{\mu_{1}-\lambda_{1}}{\mu_{1}-\lambda_{1}+\mu_{3}-\lambda_{3}}+\rho_{1} \rho_{2} \rho_{3} \frac{\mu_{1}-\lambda_{1}}{\mu_{1}-\lambda_{1}+\mu_{2}-\lambda_{2}+\mu_{3}-\lambda_{3}}} \\
& K_{3}=\frac{\rho_{3}\left(\mu_{1}-\lambda_{1}\right)}{1-\rho_{2} \frac{\mu_{1}-\lambda_{1}}{\mu_{1}-\lambda_{1}+\mu_{2}-\lambda_{2}}-\rho_{3} \frac{\mu_{1}-\lambda_{1}}{\mu_{1}-\lambda_{1}+\mu_{3}-\lambda_{3}}+\rho_{1} \rho_{2} \rho_{3} \frac{\mu_{1}-\lambda_{1}}{\mu_{1}-\lambda_{1}+\mu_{2}-\lambda_{2}+\mu_{3}-\lambda_{3}}} \\
& K_{4}=\frac{\rho_{2} \rho_{3}\left(\mu_{1}-\lambda_{1}\right)}{1-\rho_{2} \frac{\mu_{1}-\lambda_{1}}{\mu_{1}-\lambda_{1}+\mu_{2}-\lambda_{2}}-\rho_{3} \frac{\mu_{1}-\lambda_{1}}{\mu_{1}-\lambda_{1}+\mu_{3}-\lambda_{3}}+\rho_{1} \rho_{2} \rho_{3} \frac{\mu_{1}-\lambda_{1}}{\mu_{1}-\lambda_{1}+\mu_{2}-\lambda_{2}+\mu_{3}-\lambda_{3}}}
\end{aligned}
$$

Next, we provide bounds for equation (3.12), 
For a lower bound, by the definition of conditional probability,

$$
\begin{aligned}
& \int_{0+}^{\infty} \mathbb{P}\left\{M_{23}(t, s) \geq n \mid W_{2}(t)<s, W_{3}(t)<s\right\} f_{W_{1} \mid W_{1}>W_{2}, W_{1}>W_{3}}(s) d s \\
= & \int_{0+}^{\infty} \mathbb{P}\left\{M_{23}(t, s) \geq n, W_{2}(t)<s, W_{3}(t)<s\right\} \frac{f_{W_{1} \mid W_{1}>W_{2}, W_{1}>W_{3}}(s)}{\mathbb{P}\left\{W_{2}(t)<s, W_{3}(t)<s\right\}} d s \\
\geq & \int_{0+}^{\infty} \mathbb{P}\left\{M_{23}(t, s) \geq n, W_{2}(t)=0, W_{3}(t)=0\right\} f_{W_{1} \mid W_{1}>W_{2}, W_{1}>W_{3}}(s) d s \\
= & \int_{0+}^{\infty} \mathbb{P}\left\{M_{23}(t, s) \geq n, q_{2}(t)=0, q_{3}(t)=0\right\} f_{W_{1} \mid W_{1}>W_{2}, W_{1}>W_{3}}(s) d s
\end{aligned}
$$

Recall that, $q_{i}(t)$ is the size of queue $i$ at time $t$. Similarly, we also denote $q_{i j}(t)$ to be the event \{the total number of customers in queue $i$ or queue $j$ at time $t$ \}. Obviously, $q_{i j}(t)=0$ implies both $q_{i}(t)$ and $q_{j}(t)$ are 0 . The last step is true since according the definition of $W_{i}(t)=0$, which means the $i^{\text {th }}$ queue is empty. Hence, $W_{i}(t)=0$ is nothing but $q_{i}(t)=0$.

For an upper bound,

$$
\begin{aligned}
& \int_{0+}^{\infty} \mathbb{P}\left\{M_{23}(t, s) \geq n \mid W_{2}(t)<s, W_{3}(t)<s\right\} f_{W_{1} \mid W_{1}>W_{2}, W_{1}>W_{3}}(s) d s \\
= & \int_{0+}^{\infty} \mathbb{P}\left\{M_{23}(t, s) \geq n, W_{2}(t)<s, W_{3}(t)<s\right\} \frac{f_{W_{1} \mid W_{1}>W_{2}, W_{1}>W_{3}}(s)}{\mathbb{P}\left\{W_{2}(t)<s, W_{3}(t)<s\right\}} d s \\
\leq & \int_{0+}^{\infty} \mathbb{P}\left\{M_{23}(t, s) \geq n\right\} \frac{f_{W_{1} \mid W_{1}>W_{2}, W_{1}>W_{3}(s)}(s)}{\mathbb{P}\left\{W_{2}(t)=0, W_{3}(t)=0\right\}} d s \\
= & \frac{1}{\left(1-\rho_{2}\right)\left(1-\rho_{3}\right)} \int_{0+}^{\infty} \mathbb{P}\left\{M_{23}(t, s) \geq n\right\} f_{W_{1} \mid W_{1}>W_{2}, W_{1}>W_{3}}(s) d s
\end{aligned}
$$

In the last equation, we use the fact that queue 2 and queue 3 are independent and $\mathbb{P}\left\{W_{i}(t)=0\right\}=1-\rho_{i}$. To calculate the upper and lower bounds, we need to know $\mathbb{P}\left\{M_{23}(t, s) \geq n, q_{2}(t)=0, q_{3}(t)=0\right\}$ and $\mathbb{P}\left\{M_{23}(t, s) \geq n\right\}$. In the next section, 
we will focus on the computation of $\mathbb{P}\{M(T) \geq n\}$, which is the preparation for finding the upper and lower bounds.

\subsection{Computation of $\mathbb{P}\{M(T) \geq n\}$}

In this section, we consider two independent and stationary $M / M / 1$ queues with arrival rates $\lambda_{1}$ and $\lambda_{2}$ and departure rates $\mu_{1}$ and $\mu_{2}$, respectively. Suppose the capacity of each queue is infinity. We assume $\lambda_{i}<\mu_{i}$, where $i=1,2$, so that both queues are stable.

$M(t)$ is defined as the total number of those customers, who arrived at either of these two queues during interval $[0, t]$ and departed by time $t$. Let $T$ be an exponential random variable with mean $\frac{1}{\mu_{3}-\lambda_{3}}$ and $T$ is independent of those two queue processes. The density function of $T$ is $f_{T}(t)=\left(\mu_{3}-\lambda_{3}\right) e^{-\left(\mu_{3}-\lambda_{3}\right) t}$, for $t \geq 0$.

In this section, we will frequently use the following fact.

Fact 3.1 For $a>0$ and integer $k \geq 0$, we have

$$
\int_{0}^{\infty} \frac{e^{-a t} t^{k}}{k !} d t=\left(\frac{1}{a}\right)^{k+1}
$$

Proof. Using integration by parts, this can be easily checked as follows:

$$
\int_{0}^{\infty} \frac{e^{-a t} t^{k}}{k !} d t=-\left.\frac{1}{a} \frac{e^{-a t} t^{k}}{k !}\right|_{0} ^{\infty}+\frac{1}{a} \int_{0}^{\infty} \frac{e^{-a t} t^{k-1}}{(k-1) !}=\frac{1}{a} \int_{0}^{\infty} \frac{e^{-a t} t^{k-1}}{(k-1) !}
$$


CHAPTER 3. 3-M/M/1 CASE

Similarly, repeating integration by parts $k-1$ times, we will get the result.

\subsubsection{Case 1: $\mu_{1}-\lambda_{1}+\mu_{2}-\lambda_{2} \geq \mu_{3}-\lambda_{3}$}

\section{The Upper Bound}

Besides $M(t)$ defined at the beginning of this section, we also define $M^{*}(t)$ to be the total number of customers, who arrived at either one of these two queues during interval $[0, t]$. It is obvious that

$$
M(t) \leq M^{*}(t)
$$

Hence,

$$
\begin{aligned}
& \mathbb{P}\{M(T) \geq n\} \\
\leq & \mathbb{P}\left\{M^{*}(T) \geq n\right\} \\
= & \sum_{k=n}^{\infty} \int_{0}^{\infty} \mathbb{P}\left\{M^{*}(t)=k\right\} f_{T}(t) d t
\end{aligned}
$$

Since $M^{*}(t)$ is the total number of arrivals from two independent Poisson processes, $M^{*}(t)$ follows the Poisson distribution with rate $\left(\lambda_{1}+\lambda_{2}\right) t$. It gives,

$$
\begin{aligned}
& \mathbb{P}\left\{M^{*}(t)=k\right\} \\
= & t^{k} e^{-\left(\lambda_{1}+\lambda_{2}\right) t} \frac{1}{k !}\left(\lambda_{1}+\lambda_{2}\right)^{k}
\end{aligned}
$$

Combining the inequality and equation above, we have,

$$
\mathbb{P}\{M(T) \geq n\}
$$




$$
\begin{aligned}
& \leq \sum_{k=n}^{\infty} \int_{0}^{\infty} t^{k} e^{-\left(\lambda_{1}+\lambda_{2}\right) t} \frac{1}{k !}\left(\lambda_{1}+\lambda_{2}\right)^{k} f_{T}(t) d t \\
& =\sum_{k=n}^{\infty}\left(\lambda_{1}+\lambda_{2}\right)^{k}\left(\mu_{3}-\lambda_{3}\right) \int_{0}^{\infty} \frac{e^{-\left(\lambda_{1}+\lambda_{2}+\mu_{3}-\lambda_{3}\right) t} t^{k}}{k !} d t \\
& =\sum_{k=n}^{\infty}\left(\lambda_{1}+\lambda_{2}\right)^{k}\left(\mu_{3}-\lambda_{3}\right)\left(\frac{1}{\lambda_{1}+\lambda_{2}+\mu_{3}-\lambda_{3}}\right)^{k+1} \\
& =\sum_{k=n}^{\infty} \frac{\mu_{3}-\lambda_{3}}{\lambda_{1}+\lambda_{2}+\mu_{3}-\lambda_{3}}\left(\frac{\lambda_{1}+\lambda_{2}}{\lambda_{1}+\lambda_{2}+\mu_{3}-\lambda_{3}}\right)^{k} \\
& =\frac{\mu_{3}-\lambda_{3}}{\lambda_{1}+\lambda_{2}+\mu_{3}-\lambda_{3}} \frac{\left(\frac{\lambda_{1}+\lambda_{2}}{\lambda_{1}+\lambda_{2}+\mu_{3}-\lambda_{3}}\right)^{n}}{\lambda_{1}+\lambda_{2}+\lambda_{3}-\lambda_{3}} \\
& =\left(\frac{\lambda_{1}+\lambda_{2}}{\lambda_{1}+\lambda_{2}+\mu_{3}-\lambda_{3}}\right)^{n}
\end{aligned}
$$

Remark 3.1 In the above equation, the sum of infinite geometric series in the second last step is finite because $\lambda_{1}+\lambda_{2}<\lambda_{1}+\lambda_{2}+\mu_{3}-\lambda_{3}$, which implies $\frac{\lambda_{1}+\lambda_{2}}{\lambda_{1}+\lambda_{2}+\mu_{3}-\lambda_{3}}<1$

\section{The Lower Bound}

Recall the model described at the beginning of this section, we consider two independent and stationary $M / M / 1$ queues whose arrival rates are $\lambda_{1}$ and $\lambda_{2}$, and departure rates are $\mu_{1}$ and $\mu_{2}$. Now, we define the total size $q(t)$ of these two $M / M / 1$ queues at time $t$. And let $D(t)$ be the total number of departures from

either of the two queues during the interval $[0, t]$. To compute $\mathbb{P}\{M(t)=k\}$, we condition on $q(0)$, that is, condition on the total size of these two $M / M / 1$ queues 
at time 0 .

$$
\begin{aligned}
& \mathbb{P}\{M(t)=k\} \\
= & \sum_{m=0}^{\infty} \mathbb{P}\{M(t)=k \mid q(0)=m\} \mathbb{P}\{q(0)=m\} \\
\geq & \mathbb{P}\{M(t)=k \mid q(0)=0\} \mathbb{P}\{q(0)=0\} \\
= & \mathbb{P}\{D(t)=k \mid q(0)=0\} \mathbb{P}\{q(0)=0\} \\
= & \mathbb{P}\{D(t)=k \bigcap q(0)=0\} \\
\geq & C \cdot \mathbb{P}\{\tilde{D}(t)=k \bigcap \tilde{q}(0)=0\}
\end{aligned}
$$

where $\tilde{q}(t)$ is the queue size at time $t$ of $M / M / 1$ queue with arrival rate $\lambda_{1}+\lambda_{2}$ and departure rate $\mu_{1}+\mu_{1}$. $\tilde{D}(t)$ is the number of departures during the interval $[0, t]$ of this queue. $C$ is a positive constant. We can use $D(t)=k$ instead of $M(t)=k$, because, given $q(0)=0$, the number of departures during $[0, t]$ is the number of customers that arrived during the $[0, t]$ and departed by time $t$, which is the definition of $M(t)$.

Remark 3.2 In (3.19), $\mathbb{P}\{D(t)=k \bigcap q(0)=0\} \geq C \cdot \mathbb{P}\{\tilde{D}(t)=k \bigcap \tilde{q}(0)=0\}$ can be explained as follows:

The $M / M / 1$ queue with arrival rate $\lambda_{1}+\lambda_{2}$ and departure rate $\mu_{1}+\mu_{1}$ and $2-M / M / 1$ with arrival rate $\lambda_{1}, \lambda_{2}$ and departure rate $\mu_{1}, \mu_{2}$ respectively, have the same departure process. Therefore, $\tilde{D}(t)$ and $D(t)$ have the same distribution, which leads to $\mathbb{P}\{\tilde{D}(t)=k \mid \tilde{q}(0)=0\}=\mathbb{P}\{D(t)=k \mid q(0)=0\}$. 
On the other hand,

$$
\begin{aligned}
\mathbb{P}\{q(0)=0\} & =\left(1-\rho_{1}\right)\left(1-\rho_{2}\right) \\
& =\left(1-\frac{\lambda_{1}}{\mu_{1}}\right)\left(1-\frac{\lambda_{2}}{\mu_{2}}\right)>0 \\
\mathbb{P}\{\tilde{q}(0)=0\} & =(1-\rho) \\
& =\left(1-\frac{\lambda_{1}+\lambda_{2}}{\mu_{1}+\mu_{2}}\right)>0
\end{aligned}
$$

Hence, we can always find a positive constant $C$, such that

$$
\mathbb{P}\{q(0)=0\} \geq C \cdot \mathbb{P}\{\tilde{q}(0)=0\}
$$

which gives (3.19)

Now (3.19) becomes,

$$
\begin{aligned}
& \mathbb{P}\{M(t)=k\} \\
\geq & C \cdot \mathbb{P}\{\tilde{D}(t)=k \bigcap \tilde{q}(0)=0\} \\
= & C \cdot \mathbb{P}\{\tilde{D}(t)=k \mid \tilde{q}(0)=0\} \mathbb{P}\{\tilde{q}(0)=0\} \\
= & C(1-\rho) \mathbb{P}\{\tilde{D}(t)=k \mid \tilde{q}(0)=0\} \\
\geq & C(1-\rho) \mathbb{P}\{\tilde{D}(t)=k \bigcap \tilde{q}(t)=0 \mid \tilde{q}(0)=0\}
\end{aligned}
$$

From ([7]) (page 199),

$$
\mathbb{P}\{\tilde{D}(t)=k \bigcap \tilde{q}(t)=0 \mid \tilde{q}(0)=0\}
$$




$$
\begin{aligned}
& =\sum_{i=0}^{\infty} \frac{(1+i) \rho^{k}}{k !(k+i+1) !}\left[\left(\mu_{1}+\mu_{2}\right) t\right]^{2 k+i} e^{-\left(\lambda_{1}+\lambda_{2}+\mu_{1}+\mu_{2}\right) t} \\
& =\frac{\left[\left(\lambda_{1}+\lambda_{2}\right) t\right]^{k} e^{-\left(\lambda_{1}+\lambda_{2}\right) t}}{k !} \sum_{i=0}^{\infty} \frac{1+i}{(k+i+1) !}\left[\left(\mu_{1}+\mu_{2}\right) t\right]^{k+i} e^{-\left(\mu_{1}+\mu_{2}\right) t} \\
& \geq \frac{1}{k+1} \frac{\left[\left(\lambda_{1}+\lambda_{2}\right) t\right]^{k} e^{-\left(\lambda_{1}+\lambda_{2}\right) t}}{k !} \sum_{i=0}^{\infty} \frac{1}{(k+i) !}\left[\left(\mu_{1}+\mu_{2}\right) t\right]^{k+i} e^{-\left(\mu_{1}+\mu_{2}\right) t} \\
& =\frac{1}{k+1} \frac{\left[\left(\lambda_{1}+\lambda_{2}\right) t\right]^{k} e^{-\left(\lambda_{1}+\lambda_{2}\right) t}}{k !} \mathbb{P}\left\{Y_{\left(\mu_{1}+\mu_{2}\right) t} \geq k\right\}
\end{aligned}
$$

where $Y_{\left(\mu_{1}+\mu_{2}\right) t}$ is a Poisson random variable with mean $\left(\mu_{1}+\mu_{2}\right) t$.

The inequity was obtained by using the fact

$$
\frac{1+i}{(k+i+1) !}=\frac{1+i}{k+i+1} \frac{1}{(k+i) !}
$$

for any nonnegative integer, and

$$
\frac{1+i}{k+i+1} \geq \frac{1}{k+1}
$$

Now, setting $t_{0}=\frac{n}{\lambda_{1}+\lambda_{2}+\mu_{3}-\lambda_{3}}$, we have

$$
\begin{aligned}
\mathbb{P}\{M(T) \geq n\} & \geq \mathbb{P}\left\{M(T) \geq n \bigcap T \geq t_{0}\right\} \\
& \geq \mathbb{P}\left\{M\left(t_{0}\right) \geq n \bigcap T \geq t_{0}\right\} \\
& =\mathbb{P}\left\{M\left(t_{0}\right) \geq n\right\} \mathbb{P}\left\{T \geq t_{0}\right\} \\
& =\sum_{k=n}^{\infty} \mathbb{P}\left\{M\left(t_{0}\right)=k\right\} \mathbb{P}\left\{T \geq t_{0}\right\}
\end{aligned}
$$


The equation (3.22) holds because of the independence of the $2-M / M / 1$ queue process and the random variable $T$.

Combining (3.20) (3.21) and (3.23) gives

$$
\begin{aligned}
& \mathbb{P}\{M(T) \geq n\} \\
\geq & C(1-\rho) \sum_{k=n}^{\infty} \frac{1}{k+1} \frac{\left[\left(\lambda_{1}+\lambda_{2}\right) t_{0}\right]^{k} e^{-\left(\lambda_{1}+\lambda_{2}\right) t_{0}}}{k !} \mathbb{P}\left\{Y_{\left(\mu_{1}+\mu_{2}\right) t_{0}} \geq k\right\} \cdot e^{-\left(\mu_{3}-\lambda_{3}\right) t_{0}} \\
\geq & C(1-\rho) \frac{1}{n+1} \frac{\left[\left(\lambda_{1}+\lambda_{2}\right) t_{0}\right]^{n} e^{-\left(\lambda_{1}+\lambda_{2}\right) t_{0}}}{n !} \mathbb{P}\left\{Y_{\left(\mu_{1}+\mu_{2}\right) t_{0}} \geq n\right\} \cdot e^{-\left(\mu_{3}-\lambda_{3}\right) t_{0}}
\end{aligned}
$$

Since we are interested in the limiting probability of the RSQ size as $n \rightarrow \infty$, we need to find a bound for $\mathbb{P}\left\{Y_{\left(\mu_{1}+\mu_{2}\right) t_{0}} \geq n\right\}$ when $n$ goes to infinity, especially the lower bound. Evidently, this probability is greater than 0 , but 0 is not tight enough for our discussion. Next we will show $\mathbb{P}\left\{Y_{\left(\mu_{1}+\mu_{2}\right) t_{0}} \geq n\right\}$ is greater than a positive constant as $n$ goes to infinity. By definition $t_{0}=\frac{n}{\lambda_{1}+\lambda_{2}+\mu_{3}-\lambda_{3}}$ and the assumption $\mu_{1}-\lambda_{1}+\mu_{2}-\lambda_{2} \geq \mu_{3}-\lambda_{3}$, we have

$$
\left(\mu_{1}+\mu_{2}\right) t_{0}=\frac{\mu_{1}+\mu_{2}}{\lambda_{1}+\lambda_{2}+\mu_{3}-\lambda_{3}} \cdot n \geq n
$$

Let $n_{0}=\left[\left(\mu_{1}+\mu_{2}\right) t_{0}\right]$. Then, $n_{0} \geq n$. Let $X_{1}, X_{2}, \cdots, X_{n_{0}}$ be independently identical distributed Poisson random variables with mean 1. It follows from the basic property of the Poisson distribution that $X_{1}+X_{2}+\cdots+X_{n_{0}}$ is still a Poisson random variable with mean $n_{0}$. 
Thus,

$$
\begin{aligned}
& \mathbb{P}\left\{Y_{\left(\mu_{1}+\mu_{2}\right) t_{0}} \geq n\right\} \\
\geq & \mathbb{P}\left\{\frac{X_{1}+X_{2}+\cdots+X_{n_{0}}}{n_{0}} \geq \frac{n}{n_{0}}\right\} \\
\geq & \mathbb{P}\left\{\frac{X_{1}+X_{2}+\cdots+X_{n_{0}}}{n_{0}} \geq 1\right\} \\
= & \mathbb{P}\left\{\frac{X_{1}+X_{2}+\cdots+X_{n_{0}}-n_{0}}{\sqrt{n_{0}} \sqrt{n_{0}}} \geq 0\right\}
\end{aligned}
$$

By the Central Limit Theorem,

$$
\begin{aligned}
& \lim _{n_{0} \rightarrow \infty} \mathbb{P}\left\{\frac{X_{1}+X_{2}+\cdots+X_{n_{0}}-n_{0}}{\sqrt{n_{0}} \sqrt{n_{0}}} \geq 0\right\} \\
= & \int_{0}^{\infty} \frac{1}{\sqrt{2 \pi}} e^{-\frac{x^{2}}{2}} d x \\
= & \frac{1}{2}
\end{aligned}
$$

Therefore, for any $\epsilon \geq 0$, there exists some integer $N \geq 0$ such that for all $n \geq N$,

$$
\mathbb{P}\left\{Y_{\left(\mu_{1}+\mu_{2}\right) t_{0}} \geq n\right\} \geq \frac{1}{2}-\epsilon
$$

For $n \geq N$, replacing $\mathbb{P}\left\{Y_{\left(\mu_{1}+\mu_{2}\right) t_{0}} n\right\}$ by $\frac{1}{2}-\epsilon,(3.24)$ becomes,

$$
\mathbb{P}\{M(T) \geq n\} \geq C(1-\rho)\left(\frac{1}{2}-\epsilon\right) \frac{1}{n+1} \frac{\left[\left(\lambda_{1}+\lambda_{2}\right) t_{0}\right]^{n} e^{-\left(\lambda_{1}+\lambda_{2}\right) t_{0}}}{n !} e^{-\left(\mu_{3}-\lambda_{3}\right) t_{0}}
$$


By the Stirling's approximation,

$$
n !=\sqrt{2 \pi n} n^{n} e^{-n}\left(1+O\left(\frac{1}{n}\right)\right)
$$

When $n$ is sufficient large,

$$
n ! \leq \sqrt{2 \pi n} n^{n} e^{-n}
$$

Therefore, for large enough $n$, continue with (3.26),

$$
\begin{aligned}
& \mathbb{P}\{M(T) \geq n\} \\
\geq & \frac{1}{4} C(1-\rho)(1-2 \epsilon) \frac{1}{n+1} \frac{\left(\frac{\lambda_{1}+\lambda_{2}}{\lambda_{1}+\lambda_{2}+\mu_{3}-\lambda_{3}}\right)^{n} n^{n} \exp \left\{-\frac{\lambda_{1}+\lambda_{2}}{\lambda_{1}+\lambda_{2}+\mu_{3}-\lambda_{3}} n\right\}}{\sqrt{2 \pi n} n^{n} e^{-n}} \\
& \cdot \exp \left\{-\frac{\mu_{3}-\lambda_{3}}{\lambda_{1}+\lambda_{2}+\mu_{3}-\lambda_{3}} n\right\} \\
= & \frac{C(1-\rho)(1-2 \epsilon)}{4 \sqrt{2 \pi n}(n+1)}\left(\frac{\lambda_{1}+\lambda_{2}}{\lambda_{1}+\lambda_{2}+\mu_{3}-\lambda_{3}}\right)^{n}
\end{aligned}
$$

Remark 3.3 The reason of choosing $t_{0}=\frac{n}{\lambda_{1}+\lambda_{2}+\mu_{3}-\lambda_{3}}$ can be explained here.

In the case of $\mu_{1}-\lambda_{1}+\mu_{2}-\lambda_{2} \geq \mu_{3}-\lambda_{3}$, the upper bound is easy to find as shown in section 3.2.1. When we compute the upper bound, we need calculate 


$$
\begin{gathered}
\int_{0}^{\infty} \frac{e^{-\left(\lambda_{1}+\lambda_{2}\right) t}\left[\left(\lambda_{1}+\lambda_{2}\right) t\right]^{n}}{n !}\left(\mu_{3}-\lambda_{3}\right) e^{\left(\mu_{3}-\lambda_{3}\right) t} d t . \text { Suppose, as } n \text { gets large, } \\
\int_{0}^{\infty} \frac{e^{-\left(\lambda_{1}+\lambda_{2}\right) t}\left[\left(\lambda_{1}+\lambda_{2}\right) t\right]^{n}}{n !}\left(\mu_{3}-\lambda_{3}\right) e^{\left(\mu_{3}-\lambda_{3}\right) t} d t \\
\approx \max _{t \geq 0} \frac{e^{-\left(\lambda_{1}+\lambda_{2}\right) t}\left[\left(\lambda_{1}+\lambda_{2}\right) t\right]^{n}}{n !}\left(\mu_{3}-\lambda_{3}\right) e^{\left(\mu_{3}-\lambda_{3}\right) t}
\end{gathered}
$$

in some sense. It can be shown easily that the integration is maximized at,

$$
t_{0}=\frac{n}{\lambda_{1}+\lambda_{2}+\mu_{3}-\lambda_{3}}
$$

by taking the first derivative equals to 0 . So, we take $t_{0}=\frac{n}{\lambda_{1}+\lambda_{2}+\mu_{3}-\lambda_{3}}$ in the proof for the lower bound

\section{Large Deviation Result for $M(T)$ in Case 1}

From the discussion about both lower and upper bounds given above, we have already obtained that, for large $n$,

$$
\frac{C(1-\rho)(1-2 \epsilon)}{4 \sqrt{2 \pi n}(n+1)}\left(\frac{\lambda_{1}+\lambda_{2}}{\lambda_{1}+\lambda_{2}+\mu_{3}-\lambda_{3}}\right)^{n} \leq \mathbb{P}\{M(T) \geq n\} \leq\left(\frac{\lambda_{1}+\lambda_{2}}{\lambda_{1}+\lambda_{2}+\mu_{3}-\lambda_{3}}\right)^{n}
$$

Taking the logarithm and multiplying by $\frac{1}{n}$ gives,

$$
\begin{aligned}
& \frac{1}{n} \log \left\{\frac{C(1-\rho)(1-2 \epsilon)}{4 \sqrt{2 \pi n}(n+1)}\right\}+\frac{1}{n} \cdot n \log \left(\frac{\lambda_{1}+\lambda_{2}}{\lambda_{1}+\lambda_{2}+\mu_{3}-\lambda_{3}}\right) \\
\leq & \frac{1}{n} \log \mathbb{P}\{M(T) \geq n\} \leq \frac{1}{n} \cdot n \log \left(\frac{\lambda_{1}+\lambda_{2}}{\lambda_{1}+\lambda_{2}+\mu_{3}-\lambda_{3}}\right)
\end{aligned}
$$


Let the lower bound be denoted by

$$
f(n)=\frac{1}{n} \log \left\{\frac{C(1-\rho)(1-2 \epsilon)}{4 \sqrt{2 \pi n}(n+1)}\right\}+\frac{1}{n} \cdot n \log \left(\frac{\lambda_{1}+\lambda_{2}}{\lambda_{1}+\lambda_{2}+\mu_{3}-\lambda_{3}}\right),
$$

and the upper bound by

$$
g(n)=\frac{1}{n} \cdot n \log \left(\frac{\lambda_{1}+\lambda_{2}}{\lambda_{1}+\lambda_{2}+\mu_{3}-\lambda_{3}}\right)
$$

Since,

$$
\begin{aligned}
& \lim _{n \rightarrow \infty} f(n) \\
= & \lim _{n \rightarrow \infty}\left\{\frac{1}{n} \log \left\{\frac{C(1-\rho)(1-2 \epsilon)}{4 \sqrt{2 \pi n}(n+1)}\right\}+\frac{1}{n} \cdot n \log \left(\frac{\lambda_{1}+\lambda_{2}}{\lambda_{1}+\lambda_{2}+\mu_{3}-\lambda_{3}}\right)\right\} \\
= & \lim _{n \rightarrow \infty}\left\{\frac{1}{n} \log \left\{\frac{C(1-\rho)(1-2 \epsilon)}{4 \sqrt{2 \pi n}(n+1)}\right\}\right\}+\lim _{n \rightarrow \infty}\left\{\frac{1}{n} \cdot n \log \left(\frac{\lambda_{1}+\lambda_{2}}{\lambda_{1}+\lambda_{2}+\mu_{3}-\lambda_{3}}\right)\right\} \\
= & 0+\log \left\{\frac{\lambda_{1}+\lambda_{2}}{\lambda_{1}+\lambda_{2}+\mu_{3}-\lambda_{3}}\right\}=\log \left\{\frac{\lambda_{1}+\lambda_{2}}{\lambda_{1}+\lambda_{2}+\mu_{3}-\lambda_{3}}\right\}
\end{aligned}
$$

and

$$
\lim _{n \rightarrow \infty} g(n)=\lim _{n \rightarrow \infty}\left\{\frac{1}{n} \cdot n \log \left(\frac{\lambda_{1}+\lambda_{2}}{\lambda_{1}+\lambda_{2}+\mu_{3}-\lambda_{3}}\right)\right\}=\log \left\{\frac{\lambda_{1}+\lambda_{2}}{\lambda_{1}+\lambda_{2}+\mu_{3}-\lambda_{3}}\right\}
$$

by the Squeezing Theorem,

$$
\lim _{n \rightarrow \infty} \frac{1}{n} \log \mathbb{P}\{M(T) \geq n\}=\log \left\{\frac{\lambda_{1}+\lambda_{2}}{\lambda_{1}+\lambda_{2}+\mu_{3}-\lambda_{3}}\right\}
$$


CHAPTER 3. $3-M / M / 1 C A S E$

3.2.2 Case 2: $\mu_{1}-\lambda_{1}+\mu_{2}-\lambda_{2}<\mu_{3}-\lambda_{3}$

\section{The Lower Bound}

Conditioning on $T$ we get,

$$
\begin{aligned}
& \mathbb{P}\{M(T) \geq n\} \\
= & \sum_{k=n}^{\infty} \mathbb{P}\{M(T)=n\} \\
= & \int_{0}^{\infty} \sum_{k=n}^{\infty} \mathbb{P}\{M(t)=n \mid T=t\} f_{T}(t) d t
\end{aligned}
$$

Using the result of (3.20) and the $p d f$ of $T$, it becomes,

$$
\begin{aligned}
& \mathbb{P}\{M(T) \geq n\} \\
\geq & \int_{0}^{\infty} \sum_{k=n}^{\infty} C(1-\rho) \mathbb{P}\{\tilde{D}(t)=k \bigcap \tilde{q}(t)=0 \mid \tilde{q}(0)=0\}\left(\mu_{3}-\lambda_{3}\right) e^{-\left(\mu_{3}-\lambda_{3}\right) t} d t
\end{aligned}
$$

Replace $\mathbb{P}\{\tilde{D}(t)=k \bigcap \tilde{q}(t)=0 \mid \tilde{q}(0)=0\}$ by the result of (3.21) to yield,

$$
\begin{aligned}
& \mathbb{P}\{M(T) \geq n\} \\
\geq & C \frac{(1-\rho)\left(\mu_{3}-\lambda_{3}\right)}{n+1} \int_{0}^{\infty} \frac{\left[\left(\lambda_{1}+\lambda_{2}\right) t\right]^{n} e^{-\left(\lambda_{1}+\lambda_{2}\right) t}}{n !} \mathbb{P}\left\{Y_{\left(\mu_{1}+\mu_{2}\right) t}=n\right\} e^{-\left(\mu_{3}-\lambda_{3}\right) t} d t \\
= & C \frac{(1-\rho)\left(\mu_{3}-\lambda_{3}\right)}{n+1} \int_{0}^{\infty} \frac{\left[\left(\lambda_{1}+\lambda_{2}\right) t\right]^{n} e^{-\left(\lambda_{1}+\lambda_{2}\right) t}}{n !} \frac{\left[\left(\mu_{1}+\mu_{2}\right) t\right]^{n} e^{-\left(\mu_{1}+\mu_{2}\right) t}}{n !} e^{-\left(\mu_{3}-\lambda_{3}\right) t} d t \\
= & C \frac{(1-\rho)\left(\mu_{3}-\lambda_{3}\right)}{n+1} \frac{(2 n) !}{n ! n !}\left(\lambda_{1}+\lambda_{2}\right)^{n}\left(\mu_{1}+\mu_{2}\right)^{n} \int_{0}^{\infty} \frac{t^{2 n} e^{-\left(\lambda_{1}+\lambda_{2}+\mu_{1}+\mu_{2}+\mu_{3}-\lambda_{3}\right) t}}{(2 n) !} d t \\
= & C \frac{(1-\rho)\left(\mu_{3}-\lambda_{3}\right)}{n+1} \frac{(2 n) !}{n ! n !}\left(\lambda_{1}+\lambda_{2}\right)^{n}\left(\mu_{1}+\mu_{2}\right)^{n}\left(\frac{1}{\lambda_{1}+\lambda_{2}+\mu_{1}+\mu_{2}+\mu_{3}-\lambda_{3}}\right)^{2 n+1}
\end{aligned}
$$




$$
\begin{aligned}
= & C(1-\rho) \frac{\mu_{3}-\lambda_{3}}{\lambda_{1}+\lambda_{2}+\mu_{1}+\mu_{2}+\mu_{3}-\lambda_{3}} \frac{1}{n+1} \frac{(2 n) !}{n ! n !}\left(\lambda_{1}+\lambda_{2}\right)^{n}\left(\mu_{1}+\mu_{2}\right)^{n} \\
& \frac{1}{\left(\lambda_{1}+\lambda_{2}+\mu_{1}+\mu_{2}+\mu_{3}-\lambda_{3}\right)^{2 n}}
\end{aligned}
$$

where the integral $\int_{0}^{\infty} \frac{t^{2 n} e^{-\left(\lambda_{1}+\lambda_{2}+\mu_{1}+\mu_{2}+\mu_{3}-\lambda_{3}\right) t}}{(2 n) !} d t$ was evaluated by applying Fact (3.1)

By the Stirling's approximation, for large enough $n$,

$$
\begin{aligned}
\frac{(2 n) !}{n ! n !} & =\frac{\sqrt{4 \pi n}(2 n)^{2 n} e^{-2 n}(1+O(1 / n))}{\left[\sqrt{2 \pi n}(n)^{n} e^{-n}(1+O(1 / n))\right]^{2}} \\
& \geq \frac{C_{1}}{\sqrt{n}} 4^{n}
\end{aligned}
$$

for some positive constant $C_{1}$.

Hence, (3.33) can be written as,

$$
\begin{aligned}
& \mathbb{P}\{M(T) \geq n\} \\
\geq & \frac{C C_{1}(1-\rho)\left(\mu_{3}-\lambda_{3}\right)}{\lambda_{1}+\lambda_{2}+\mu_{1}+\mu_{2}+\mu_{3}-\lambda_{3}} \frac{1}{\sqrt{n}(n+1)}\left(\frac{4\left(\lambda_{1}+\lambda_{2}\right)\left(\mu_{1}+\mu_{2}\right)}{\left(\lambda_{1}+\lambda_{2}+\mu_{1}+\mu_{2}+\mu_{3}-\lambda_{3}\right)^{2}}\right)^{n} \\
= & M_{1} \frac{1}{\sqrt{n}(n+1)}\left(\frac{4\left(\lambda_{1}+\lambda_{2}\right)\left(\mu_{1}+\mu_{2}\right)}{\left(\lambda_{1}+\lambda_{2}+\mu_{1}+\mu_{2}+\mu_{3}-\lambda_{3}\right)^{2}}\right)^{n}
\end{aligned}
$$

where $M_{1}$ is a positive constant by combining the constant items in the equation which does not depend on $n$. 


\section{The Upper Bound}

$$
\begin{aligned}
& \mathbb{P}\{M(t) \geq n\} \\
\leq & \mathbb{P}\{\text { at least } n \text { customers arrived to the } 2-M / M / 1 \text { system during the } \\
& \text { interval }[0, t], \text { and at least } n \text { customers are served on the same interval }\} \\
\leq & \sum_{k=n}^{\infty} \mathbb{P}\{k \text { customers arrived on interval }[0, t]\} \\
& \sum_{m=1}^{n} \mathbb{P}\left\{\sum_{i=1}^{m} X_{1 i} \leq t\right\} \mathbb{P}\left\{\sum_{j=m+1}^{n} Y_{2 j} \leq t\right\} \\
= & \sum_{k=n}^{\infty} \mathbb{P}\left\{M^{*}(t)=k\right\}\left[\sum_{m=1}^{n} \mathbb{P}\left\{\sum_{i=1}^{m} X_{1 i} \leq t\right\} \mathbb{P}\left\{\sum_{j=m+1}^{n} Y_{2 j} \leq t\right\}\right]
\end{aligned}
$$

where $X_{1 i}^{\prime} s$ and $Y_{2 j}^{\prime} s$ are independent service times of queue 1 and queue 2 respectively.

By basic properties of the Poisson random variable, We have

$$
\begin{aligned}
& \mathbb{P}\left\{M^{*}(t)=k\right\} \\
= & \frac{\left[\left(\lambda_{1}+\lambda_{2}\right) t\right]^{k} e^{-\left(\lambda_{1}+\lambda_{2}\right) t}}{k !}
\end{aligned}
$$

As discussed before, the departure process of the $M / M / 1$ queue with arrival rate $\lambda_{1}+\lambda_{2}$ and service rate $\mu_{1}+\mu_{2}$, and the departure process from two independent 
$M / M / 1$ queues have the same distribution. Hence,

$$
\begin{aligned}
& \sum_{m=1}^{n} \mathbb{P}\left\{\sum_{i=1}^{m} X_{1 i} \leq t\right\} \mathbb{P}\left\{\sum_{j=m+1}^{n} Y_{2 j} \leq t\right\} \\
= & \mathbb{P}\{D(t) \geq n\} \\
= & \mathbb{P}\{\tilde{D}(t) \geq n\} \\
= & \mathbb{P}\left\{\sum_{m=1}^{n} Z_{m} \leq t\right\}
\end{aligned}
$$

where $Z_{1}, Z_{2}, \cdots Z_{n}$ are i.i.d. service times of $M / M / 1$ queue with arrival rate $\lambda_{1}+\lambda_{2}$ and service rate $\mu_{1}+\mu_{2}$, which are i.i.d. exponential distributions with rate $\mu_{1}+\mu_{2}$. It is a standard result that $\sum_{m=1}^{n} Z_{i}$ has a Gamma distribution with the probability density function,

$$
f(t)=\frac{\left(\mu_{1}+\mu_{2}\right) e^{-\left(\mu_{1}+\mu_{2}\right) t}\left[\left(\mu_{1}+\mu_{2}\right) t\right]^{n-1}}{(n-1) !}
$$

Hence, combining all the results above gives

$$
\begin{aligned}
& \mathbb{P}\{M(T) \geq n\}=\sum_{k=n}^{\infty} \mathbb{P}\{M(T)=n\} \\
= & \int_{0}^{\infty} \sum_{k=n}^{\infty} \mathbb{P}\{M(t)=n \mid T=t\} f_{T}(t) d t \\
\leq & \sum_{k=n}^{\infty} \int_{0}^{\infty} \mathbb{P}\left\{M^{*}(t)=k\right\} \mathbb{P}\left\{\sum_{m=1}^{n} Z_{m} \leq t\right\} f_{T}(t) d t \\
= & \sum_{k=n}^{\infty} \int_{0}^{\infty} \frac{\left[\left(\lambda_{1}+\lambda_{2}\right) t\right]^{k} e^{-\left(\lambda_{1}+\lambda_{2}\right) t}}{k !} \int_{0}^{t} \frac{\left(\mu_{1}+\mu_{2}\right) e^{-\left(\mu_{1}+\mu_{2}\right) \tau}\left[\left(\mu_{1}+\mu_{2}\right) \tau\right]^{n-1}}{(n-1) !} d \tau \\
& \cdot\left(\mu_{3}-\lambda_{3}\right) e^{-\left(\mu_{3}-\lambda_{3}\right) t} d t
\end{aligned}
$$




$$
\begin{aligned}
= & \left(\mu_{1}+\mu_{2}\right)\left(\mu_{3}-\lambda_{3}\right) \sum_{k=n}^{\infty} \int_{0}^{\infty} \int_{\tau}^{\infty} \frac{e^{-\left(\lambda_{1}+\lambda_{2}+\mu_{3}-\lambda_{3}\right) t}\left[\left(\lambda_{1}+\lambda_{2}\right) t\right]^{k}}{k !} d t \\
& . \frac{e^{-\left(\mu_{1}+\mu_{2}\right) \tau}\left[\left(\mu_{1}+\mu_{2}\right) \tau\right]^{n-1}}{(n-1) !} d \tau
\end{aligned}
$$

In the last step, the order of the integrals was interchanged.

Let $t=\tau+u,(3.39)$ becomes,

$$
\begin{aligned}
& \mathbb{P}\{M(T) \geq n\} \\
\leq & \left(\mu_{1}+\mu_{2}\right)\left(\mu_{3}-\lambda_{3}\right) \sum_{k=n}^{\infty} \int_{0}^{\infty} \int_{0}^{\infty} \frac{e^{-\left(\lambda_{1}+\lambda_{2}+\mu_{3}-\lambda_{3}\right)(\tau+u)}\left[\left(\lambda_{1}+\lambda_{2}\right)(\tau+u)\right]^{k}}{k !} d u \\
& . \frac{e^{-\left(\mu_{1}+\mu_{2}\right) \tau}\left[\left(\mu_{1}+\mu_{2}\right) \tau\right]^{n-1}}{(n-1) !} d \tau \\
= & \left(\mu_{1}+\mu_{2}\right)\left(\mu_{3}-\lambda_{3}\right) \sum_{k=n}^{\infty}\left(\lambda_{1}+\lambda_{2}\right)^{k} \int_{0}^{\infty} \int_{0}^{\infty} \frac{e^{-\left(\lambda_{1}+\lambda_{2}+\mu_{3}-\lambda_{3}\right) u} \sum_{i=0}^{k} \frac{k ! u^{i} \tau^{k-i}}{i !(k-i) !}}{k !} d u \\
& \cdot \frac{e^{-\left(\lambda_{1}+\lambda_{2}+\mu_{1}+\mu_{2}+\mu_{3}-\lambda_{3}\right) \tau}\left[\left(\mu_{1}+\mu_{2}\right) \tau\right]^{n-1}}{(n-1) !} d \tau \\
= & \left(\mu_{1}+\mu_{2}\right)\left(\mu_{3}-\lambda_{3}\right) \sum_{k=n}^{\infty}\left(\lambda_{1}+\lambda_{2}\right)^{k} \sum_{i=0}^{k} \frac{1}{(k-i) !} \int_{0}^{\infty} \int_{0}^{\infty} \frac{e^{-\left(\lambda_{1}+\lambda_{2}+\mu_{3}-\lambda_{3}\right) u} u^{i}}{i !} d u \\
& . \frac{e^{-\left(\lambda_{1}+\lambda_{2}+\mu_{1}+\mu_{2}+\mu_{3}-\lambda_{3}\right) \tau} \tau^{k-i}\left[\left(\mu_{1}+\mu_{2}\right) \tau\right]^{n-1}}{(n-1) !} d \tau \\
= & \left(\mu_{1}+\mu_{2}\right)^{n}\left(\mu_{3}-\lambda_{3}\right) \sum_{k=n}^{\infty}\left(\lambda_{1}+\lambda_{2}\right)^{k} \sum_{i=0}^{k} \frac{1}{(k-i) !} \frac{1}{\left(\lambda_{1}+\lambda_{2}+\mu_{3}-\lambda_{3}\right)^{i+1}} \\
& \cdot \int_{0}^{\infty} \frac{e^{-\left(\lambda_{1}+\lambda_{2}+\mu_{1}+\mu_{2}+\mu_{3}-\lambda_{3}\right) \tau} \tau^{n-1+k-i}}{(n-1) !} d \tau
\end{aligned}
$$




$$
\begin{aligned}
= & \left(\mu_{1}+\mu_{2}\right)^{n}\left(\mu_{3}-\lambda_{3}\right) \sum_{k=n}^{\infty}\left(\lambda_{1}+\lambda_{2}\right)^{k} \sum_{i=0}^{k} \frac{(n-1+k-i) !}{(k-i) !(n-1) !} \frac{1}{\left(\lambda_{1}+\lambda_{2}+\mu_{3}-\lambda_{3}\right)^{i+1}} \\
& \cdot \frac{1}{\left(\lambda_{1}+\lambda_{2}+\mu_{1}+\mu_{2}+\mu_{3}-\lambda_{3}\right)^{n+k-i}} \\
= & \frac{\mu_{3}-\lambda_{3}}{\lambda_{1}+\lambda_{2}+\mu_{3}-\lambda_{3}}\left(\frac{\mu_{1}+\mu_{2}}{\lambda_{1}+\lambda_{2}+\mu_{1}+\mu_{2}+\mu_{3}-\lambda_{3}}\right)^{n} \\
& \cdot \sum_{k=n}^{\infty}\left(\frac{\lambda_{1}+\lambda_{2}}{\lambda_{1}+\lambda_{2}+\mu_{1}+\mu_{2}+\mu_{3}-\lambda_{3}}\right)^{k} \\
& \cdot \sum_{i=0}^{k} \frac{(n-1+k-i) !}{(k-i) !(n-1) !}\left(\frac{\lambda_{1}+\lambda_{2}+\mu_{1}+\mu_{2}+\mu_{3}-\lambda_{3}}{\lambda_{1}+\lambda_{2}+\mu_{3}-\lambda_{3}}\right)^{i}
\end{aligned}
$$

where the integrals were computed by using Fact 3.1.

For $i=0,1 \cdots k$, let

$$
\alpha(k, i)=\frac{(n-1+k-i) !}{2^{k}(k-i) !(n-1) !}
$$

and

$$
\beta=\frac{\lambda_{1}+\lambda_{2}+\mu_{1}+\mu_{2}+\mu_{3}-\lambda_{3}}{\lambda_{1}+\lambda_{2}+\mu_{3}-\lambda_{3}}
$$

Under the assumption made in this section: $\mu_{1}-\lambda_{1}+\mu_{2}-\lambda_{2}<\mu_{3}-\lambda_{3}$ implies $\beta<2$. Note that

$$
\frac{\alpha(k+1, i)}{\alpha(k, i)}=\frac{n-k-i}{2(k+1-i)}=\frac{1+\frac{n-1}{k+1-i}}{2}
$$


Then, for each fixed $i \in\{0,1,2, \cdots, k\}$,

$$
\frac{\alpha(k+1, i)}{\alpha(k, i)} \begin{cases}\geq 1, & \text { if } k \leq n+i-2 \\ <1, & \text { if } k>n+i-2\end{cases}
$$

From the inequality above, it is clear that $\alpha(k, i)$ increases in $k$ in the interval $(0, n+i-2) ; \alpha(k+1, i)=\alpha(k, i)$, when $k=n+i-2$; and decreases for $k>n+i-2$. Therefore, $\alpha(k, i)$ is maximized at $k=n+i-2$ and $k=n+i-1$ for each fixed $i$. In our proof, we choose $k=n+i-1$, and have

$$
\alpha(n+i-1, i)=\frac{(2 n-2) !}{(n-1) !(n-1) ! 2^{n+i-1}}
$$

Hence, the sum in (3.40) indexed by $i$ becomes,

$$
\begin{aligned}
& \sum_{i=0}^{k} \frac{(n-1+k-i) !}{(k-i) !(n-1) !}\left(\frac{\lambda_{1}+\lambda_{2}+\mu_{1}+\mu_{2}+\mu_{3}-\lambda_{3}}{\lambda_{1}+\lambda_{2}+\mu_{3}-\lambda_{3}}\right)^{i} \\
= & 2^{k} \sum_{i=0}^{k} \alpha(k, i) \beta^{i} \\
\leq & 2^{k-n} \sum_{i=0}^{k} \frac{2(2 n-2) !}{(n-1) !(n-1) !}\left(\frac{\beta}{2}\right)^{i} \\
\leq & 2^{k-n} \frac{2(2 n-2) !}{(n-1) !(n-1) !} \sum_{i=0}^{\infty}\left(\frac{\beta}{2}\right)^{i} \\
= & 2^{k-n} \frac{2(2 n-2) !}{(n-1) !(n-1) !} \frac{2}{2-\beta}
\end{aligned}
$$

where $\sum_{i=0}^{\infty}\left(\frac{\beta}{2}\right)^{i}$ is finite, since $\beta<2$ implies $\frac{\beta}{2}<1$. 
Combining (3.40) and (3.44), an upper bound of $\mathbb{P}\{M(T) \geq n\}$ is given by

$$
\begin{aligned}
& \mathbb{P}\{M(T) \geq n\} \\
\leq & \frac{4\left(\mu_{3}-\lambda_{3}\right)}{(2-\beta)\left(\lambda_{1}+\lambda_{2}+\mu_{3}-\lambda_{3}\right)} \frac{(2 n-2) !}{2^{n}(n-1) !(n-1) !}\left(\frac{\mu_{1}+\mu_{2}}{\lambda_{1}+\lambda_{2}+\mu_{1}+\mu_{2}+\mu_{3}-\lambda_{3}}\right)^{n} \\
& \cdot \sum_{k=n}^{\infty}\left(\frac{2\left(\lambda_{1}+\lambda_{2}\right)}{\lambda_{1}+\lambda_{2}+\mu_{1}+\mu_{2}+\mu_{3}-\lambda_{3}}\right)^{k} \\
= & \frac{4\left(\mu_{3}-\lambda_{3}\right)}{\mu_{3}-\lambda_{3}-\left(\mu_{1}+\mu_{2}\right)+\left(\lambda_{1}+\lambda_{2}\right)} \frac{(2 n-2) !}{2^{n}(n-1) !(n-1) !} \\
& \cdot\left(\frac{\mu_{1}+\mu_{2}}{\lambda_{1}+\lambda_{2}+\mu_{1}+\mu_{2}+\mu_{3}-\lambda_{3}}\right)^{n} \frac{\left(\frac{2\left(\lambda_{1}+\lambda_{2}\right)}{\lambda_{1}+\lambda_{2}+\mu_{1}+\mu_{2}+\mu_{3}-\lambda_{3}}\right)^{n}}{2\left(\lambda_{1}+\lambda_{2}\right)} \\
&
\end{aligned}
$$

In the last equation, the sum is finite because $\mu_{1}-\lambda_{1}+\mu_{2}-\lambda_{2}<\mu_{3}-\lambda_{3}$, $\lambda_{1}<\mu_{1}$ and $\lambda_{2}<\mu_{2}$, which implies $\frac{2\left(\lambda_{1}+\lambda_{2}\right)}{\lambda_{1}+\lambda_{2}+\mu_{1}+\mu_{2}+\mu_{3}-\lambda_{3}}<1$

Stirling's approximation gives, for large enough $n$,

$$
\begin{aligned}
\frac{(2 n) !}{n ! n !} & =\frac{\sqrt{4 \pi n}(2 n)^{2} n e^{-2 n}(1+O(1 / n))}{\left[\sqrt{2 \pi n}(n)^{n} e^{-n}(1+O(1 / n))\right]^{2}} \\
& \leq \frac{C_{2}}{\sqrt{n}} 4^{n}
\end{aligned}
$$

for some positive constant $C_{2}$.

Therefore,

$$
\mathbb{P}\{M(T) \geq n\} \leq \frac{M_{2}}{\sqrt{n}}\left(\frac{4\left(\lambda_{1}+\lambda_{2}\right)\left(\mu_{1}+\mu_{2}\right)}{\left(\lambda_{1}+\lambda_{2}+\mu_{1}+\mu_{2}+\mu_{3}-\lambda_{3}\right)^{2}}\right)^{n}
$$


where $M_{2}$ is given by combining the constant items in the equation which do not depend on $n$.

Remark 3.4 In case 2, the upper bound $\mathbb{P}\{M(t) \geq n\} \leq \mathbb{P}\{$ at least $n$ customers arrived at the system during the interval $[0, t]$, and at least $n$ customers are served on the same interval\} is used. But in the case $\mu_{1}-\lambda_{1}+$ $\mu_{2}-\lambda_{2} \geq \mu_{3}-\lambda_{3}$, a different inequality $\mathbb{P}\{M(T) \geq n\} \leq \mathbb{P}\left\{M^{*}(T) \geq n\right\}$ is used, where $M^{*}(t)=\{$ the total number of customers, who arrived at either of these two queues during interval $[0, t]\}$. To see why it is necessary, consider the integral in the derivation for the lower bound.

In equation (3.33), we need to evaluate

$$
\int_{0}^{\infty} \frac{\left[\left(\lambda_{1}+\lambda_{2}\right) t\right]^{n} e^{-\left(\lambda_{1}+\lambda_{2}\right) t}}{n !} \frac{\left[\left(\mu_{1}+\mu_{2}\right) t\right]^{n} e^{-\left(\mu_{1}+\mu_{2}\right) t}}{n !} e^{-\left(\mu_{3}-\lambda_{3}\right) t} d t
$$

As discussed in remark (3.3), suppose, as $n$ gets large,

$$
\begin{aligned}
& \int_{0}^{\infty} \frac{\left[\left(\lambda_{1}+\lambda_{2}\right) t\right]^{n} e^{-\left(\lambda_{1}+\lambda_{2}\right) t}}{n !} \frac{\left[\left(\mu_{1}+\mu_{2}\right) t\right]^{n} e^{-\left(\mu_{1}+\mu_{2}\right) t}}{n !} e^{-\left(\mu_{3}-\lambda_{3}\right) t} d t \\
\approx & \max _{t \geq 0} \frac{e^{-\left(\lambda_{1}+\lambda_{2}\right) t}\left[\left(\lambda_{1}+\lambda_{2}\right)^{n}\right]}{n !} \frac{e^{-\left(\mu_{1}+\mu_{2}\right) t}\left[\left(\mu_{1}+\mu_{2}\right) t\right]^{n}}{n !} e^{-\left(\mu_{3}-\lambda_{3}\right) t}
\end{aligned}
$$

in some sense. It can be shown easily that the integral is maximized at, $t_{0}=\frac{2 n}{\lambda_{1}+\lambda_{2}+\mu_{1}+\mu_{2}+\mu_{3}-\lambda_{3}}$.

Note that when $\mu_{1}-\lambda_{1}+\mu_{2}-\lambda_{2}<\mu_{3}-\lambda_{3}$,

$$
\left(\mu_{1}+\mu_{2}\right) t_{0}=\frac{2\left(\mu_{1}+\mu_{2}\right) n}{\lambda_{1}+\lambda_{2}+\mu_{1}+\mu_{2}+\mu_{3}-\lambda_{3}}<n
$$


For the lower bound in Case 2, the method used in Subsection 3.2.1 to show that $\mathbb{P}\left\{Y_{\left(\mu_{1}+\mu_{2}\right) t_{0}} \geq n\right\}$ is greater than a positive constant (as $n$ goes to infinity) does not work any more. Therefore, we need to find a tighter upper bound in this case for $\mathbb{P}\left\{M^{*}(T) \geq n\right\}$ rather than only looking at the arrival process.

\section{Large Deviation Result for $M(T)$ in Case 2}

From the discussion above, we already obtain that, for large n,

$$
\begin{aligned}
& \frac{M_{1}}{\sqrt{n}(n+1)}\left(\frac{4\left(\lambda_{1}+\lambda_{2}\right)\left(\mu_{1}+\mu_{2}\right)}{\left(\lambda_{1}+\lambda_{2}+\mu_{1}+\mu_{2}+\mu_{3}-\lambda_{3}\right)^{2}}\right)^{n} \leq \mathbb{P}\{M(T) \geq n\} \\
\leq & \frac{M_{2}}{\sqrt{n}}\left(\frac{4\left(\lambda_{1}+\lambda_{2}\right)\left(\mu_{1}+\mu_{2}\right)}{\left(\lambda_{1}+\lambda_{2}+\mu_{1}+\mu_{2}+\mu_{3}-\lambda_{3}\right)^{2}}\right)^{n}
\end{aligned}
$$

Taking the logarithm and multiplying by $\frac{1}{n}$, leads to,

$$
\begin{aligned}
& \frac{1}{n} \log \left\{\frac{M_{1}}{\sqrt{n}(n+1)}\right\}+\frac{1}{n} \cdot n \log \left\{\frac{4\left(\lambda_{1}+\lambda_{2}\right)\left(\mu_{1}+\mu_{2}\right)}{\left(\lambda_{1}+\lambda_{2}+\mu_{1}+\mu_{2}+\mu_{3}-\lambda_{3}\right)^{2}}\right\} \\
\leq & \frac{1}{n} \log \mathbb{P}\{M(T) \geq n\} \\
\leq & \frac{1}{n} \log \left\{\frac{M_{2}}{\sqrt{n}}\right\}+\frac{1}{n} \cdot n \log \left\{\frac{4\left(\lambda_{1}+\lambda_{2}\right)\left(\mu_{1}+\mu_{2}\right)}{\left(\lambda_{1}+\lambda_{2}+\mu_{1}+\mu_{2}+\mu_{3}-\lambda_{3}\right)^{2}}\right\}
\end{aligned}
$$

Let the lower bound be denoted by

$$
f(n)=\frac{1}{n} \log \left\{\frac{M_{1}}{\sqrt{n}(n+1)}\right\}+\frac{1}{n} \cdot n \log \left\{\frac{4\left(\lambda_{1}+\lambda_{2}\right)\left(\mu_{1}+\mu_{2}\right)}{\left(\lambda_{1}+\lambda_{2}+\mu_{1}+\mu_{2}+\mu_{3}-\lambda_{3}\right)^{2}}\right\}
$$

and the upper bound by

$$
g(n)=\frac{1}{n} \log \left\{\frac{M_{2}}{\sqrt{n}}\right\}+\frac{1}{n} \cdot n \log \left\{\frac{4\left(\lambda_{1}+\lambda_{2}\right)\left(\mu_{1}+\mu_{2}\right)}{\left(\lambda_{1}+\lambda_{2}+\mu_{1}+\mu_{2}+\mu_{3}-\lambda_{3}\right)^{2}}\right\} .
$$


Compute limit of $f(n)$, we have,

$$
\begin{aligned}
& \lim _{n \rightarrow \infty} f(n) \\
= & \lim _{n \rightarrow \infty}\left\{\frac{1}{n} \log \left\{\frac{M_{1}}{\sqrt{n}(n+1)}\right\}+\frac{1}{n} \cdot n \log \left\{\frac{4\left(\lambda_{1}+\lambda_{2}\right)\left(\mu_{1}+\mu_{2}\right)}{\left(\lambda_{1}+\lambda_{2}+\mu_{1}+\mu_{2}+\mu_{3}-\lambda_{3}\right)^{2}}\right\}\right\} \\
= & \lim _{n \rightarrow \infty}\left\{\frac{1}{n} \log \left\{\frac{M_{1}}{\sqrt{n}(n+1)}\right\}\right\}+\lim _{n \rightarrow \infty}\left\{\log \left\{\frac{4\left(\lambda_{1}+\lambda_{2}\right)\left(\mu_{1}+\mu_{2}\right)}{\left(\lambda_{1}+\lambda_{2}+\mu_{1}+\mu_{2}+\mu_{3}-\lambda_{3}\right)^{2}}\right\}\right\} \\
= & 0+\log \left\{\frac{4\left(\lambda_{1}+\lambda_{2}\right)\left(\mu_{1}+\mu_{2}\right)}{\left(\lambda_{1}+\lambda_{2}+\mu_{1}+\mu_{2}+\mu_{3}-\lambda_{3}\right)^{2}}\right\} \\
= & \log \left\{\frac{4\left(\lambda_{1}+\lambda_{2}\right)\left(\mu_{1}+\mu_{2}\right)}{\left(\lambda_{1}+\lambda_{2}+\mu_{1}+\mu_{2}+\mu_{3}-\lambda_{3}\right)^{2}}\right\}
\end{aligned}
$$

Similarly,

$$
\lim _{n \rightarrow \infty} g(n)=\log \left\{\frac{4\left(\lambda_{1}+\lambda_{2}\right)\left(\mu_{1}+\mu_{2}\right)}{\left(\lambda_{1}+\lambda_{2}+\mu_{1}+\mu_{2}+\mu_{3}-\lambda_{3}\right)^{2}}\right\}
$$

By the Squeezing Theorem,

$$
\lim _{n \rightarrow \infty} \frac{1}{n} \log \mathbb{P}\{M(T) \geq n\}=\log \left\{\frac{4\left(\lambda_{1}+\lambda_{2}\right)\left(\mu_{1}+\mu_{2}\right)}{\left(\lambda_{1}+\lambda_{2}+\mu_{1}+\mu_{2}+\mu_{3}-\lambda_{3}\right)^{2}}\right\}
$$

\subsubsection{Large Deviation Results for $M(T)$}

Combining the results in Section 3.2.1 and Section 3.2.2, we summarize the main result of this section as follows: 


\section{Theorem 3.1}

$$
\begin{aligned}
& \lim _{n \rightarrow \infty} \frac{1}{n} \log \mathbb{P}\{M(T) \geq n\} \\
= & \begin{cases}\log \left\{\frac{\lambda_{1}+\lambda_{2}}{\lambda_{1}+\lambda_{2}+\mu_{3}-\lambda_{3}}\right\}, & \text { if } \mu_{1}-\lambda_{1}+\mu_{2}-\lambda_{2} \geq \mu_{3}-\lambda_{3} \\
\log \left\{\frac{4\left(\lambda_{1}+\lambda_{2}\right)\left(\mu_{1}+\mu_{2}\right)}{\left(\lambda_{1}+\lambda_{2}+\mu_{1}+\mu_{2}+\mu_{3}-\lambda_{3}\right)^{2}}\right\}, & \text { if } \mu_{1}-\lambda_{1}+\mu_{2}-\lambda_{2}<\mu_{3}-\lambda_{3}\end{cases}
\end{aligned}
$$

\section{Lemma 3.1}

$$
\begin{aligned}
& \lim _{n \rightarrow \infty} \frac{1}{n} \log \mathbb{P}\{M(T) \geq n \bigcap q(T)=0\} \\
& \geq \begin{cases}\log \left\{\frac{\lambda_{1}+\lambda_{2}}{\lambda_{1}+\lambda_{2}+\mu_{3}-\lambda_{3}}\right\}, & \text { if } \mu_{1}-\lambda_{1}+\mu_{2}-\lambda_{2} \geq \mu_{3}-\lambda_{3} \\
\log \left\{\frac{4\left(\lambda_{1}+\lambda_{2}\right)\left(\mu_{1}+\mu_{2}\right)}{\left(\lambda_{1}+\lambda_{2}+\mu_{1}+\mu_{2}+\mu_{3}-\lambda_{3}\right)^{2}}\right\}, & \text { if } \mu_{1}-\lambda_{1}+\mu_{2}-\lambda_{2}<\mu_{3}-\lambda_{3}\end{cases}
\end{aligned}
$$

Proof. This is an intermediate step in the proof for the lower bound in Theorem

\section{1}

\subsection{Conclusion}

In this section, we will combine the results of the previous two sections and prove the main theorem. Without the loss of generality, we only provide details for the case $\mu_{1}-\lambda_{1} \leq \mu_{2}-\lambda_{2} \leq \mu_{3}-\lambda_{3}$ and $\mu_{1}-\lambda_{1}+\mu_{2}-\lambda_{2} \leq \mu_{3}-\lambda_{3}$. Other cases can be discussed in exactly the same way. 


\section{Theorem 3.2 (Main Result 1)}

$$
\begin{aligned}
& \lim _{n \rightarrow \infty} \frac{1}{n} \log \mathbb{P}\left\{q^{r}(t) \geq n\right\} \\
= & \max \left\{\log \left\{\frac{\lambda_{2}+\lambda_{3}}{\lambda_{2}+\lambda_{3}+\mu_{1}-\lambda_{1}}\right\}, \log \left\{\frac{\lambda_{1}+\lambda_{3}}{\lambda_{1}+\lambda_{3}+\mu_{2}-\lambda_{2}}\right\},\right. \\
& \left.\log \left\{\frac{4\left(\lambda_{1}+\lambda_{3}\right)\left(\mu_{1}+\mu_{3}\right)}{\left(\lambda_{1}+\mu_{1}+2 \mu_{3}+\mu_{2}-\lambda_{2}\right)^{2}}\right\}, \log \left\{\frac{4\left(\lambda_{1}+\lambda_{2}\right)\left(\mu_{1}+\mu_{2}\right)}{\left(\lambda_{1}+\lambda_{2}+\mu_{1}+\mu_{2}+\mu_{3}-\lambda_{3}\right)^{2}}\right\}\right\}
\end{aligned}
$$

Proof. Start with (3.16)

$$
\begin{aligned}
& \int_{0+}^{\infty} \mathbb{P}\left\{M_{23}(t, s) \geq n \mid W_{2}(t)<s, W_{3}(t)<s\right\} f_{W_{1} \mid W_{1}>W_{2}, W_{1}>W_{3}}(s) d s \\
\geq & \int_{0+}^{\infty} \mathbb{P}\left\{M_{23}(t, s) \geq n, q_{2}(t)=0, q_{3}(t)=0\right\} f_{W_{1} \mid W_{1}>W_{2}, W_{1}>W_{3}}(s) d s \\
= & \int_{0+}^{\infty} \mathbb{P}\left\{M_{23}(s) \geq n, q_{23}(s)=0\right\} f_{W_{1} \mid W_{1}>W_{2}, W_{1}>W_{3}}(s) d s \\
= & K_{1} \int_{0}^{\infty} \mathbb{P}\left\{M_{23}(s) \geq n, q_{23}(s)=0\right\} e^{-\left(\mu_{1}-\lambda_{1}\right) s} d s \\
& -K_{2} \int_{0}^{\infty} \mathbb{P}\left\{M_{23}(s) \geq n, q_{23}(s)=0\right\} e^{-\left(\mu_{1}-\lambda_{1}+\mu_{2}-\lambda_{2}\right) s} d s \\
& -K_{3} \int_{0}^{\infty} \mathbb{P}\left\{M_{23}(s) \geq n, q_{23}(s)=0\right\} e^{-\left(\mu_{1}-\lambda_{1}+\mu_{3}-\lambda_{3}\right) s} d s \\
& +K_{4} \int_{0}^{\infty} \mathbb{P}\left\{M_{23}(s) \geq n, q_{23}(s)=0\right\} e^{-\left(\mu_{1}-\lambda_{1}+\mu_{2}-\lambda_{2}+\mu_{3}-\lambda_{3}\right) s} d s
\end{aligned}
$$

Where $K_{1}, K_{2}, K_{3}$ and $K_{4}$ are positive constants and independent with queue size $n$. In the above equation, we use the fact that the condition density of $W_{1}$ given $\left\{W_{1}>W_{2}, W_{1}>W_{3}\right\}$ takes the form as in (3.15). 
For the first term $K_{1} \int_{0}^{\infty} \mathbb{P}\left\{M_{23}(s) \geq n, q_{23}(s)=0\right\} e^{-\left(\mu_{1}-\lambda_{1}\right) s} d s$ in (3.52).

By Lemma (3.1)

$$
\begin{aligned}
& \lim _{n \rightarrow \infty} \frac{1}{n} \log \int_{0}^{\infty} \mathbb{P}\left\{M_{23}(s) \geq n, q_{23}(s)=0\right\} e^{-\left(\mu_{1}-\lambda_{1}\right) s} d s \\
\geq & \begin{cases}\log \left\{\frac{\lambda_{2}+\lambda_{3}}{\lambda_{2}+\lambda_{3}+\mu_{1}-\lambda_{1}}\right\}, & \text { if } \mu_{2}-\lambda_{2}+\mu_{3}-\lambda_{3} \geq \mu_{1}-\lambda_{1} \\
\log \left\{\frac{4\left(\lambda_{2}+\lambda_{3}\right)\left(\mu_{2}+\mu_{3}\right)}{\left(\lambda_{2}+\lambda_{3}+\mu_{2}+\mu_{3}+\mu_{1}-\lambda_{1}\right)^{2}}\right\}, & \text { if } \mu_{2}-\lambda_{2}+\mu_{3}-\lambda_{3}<\mu_{1}-\lambda_{1}\end{cases}
\end{aligned}
$$

From the assumption $\mu_{1}-\lambda_{1} \leq \mu_{2}-\lambda_{2} \leq \mu_{3}-\lambda_{3}$ and $\mu_{1}-\lambda_{1}+\mu_{2}-\lambda_{2} \leq \mu_{3}-\lambda_{3}$, we can get $\mu_{2}-\lambda_{2}+\mu_{3}-\lambda_{3} \geq \mu_{1}-\lambda_{1}$. Hence,

$$
\begin{aligned}
& \lim _{n \rightarrow \infty} \frac{1}{n} \log \int_{0}^{\infty} \mathbb{P}\left\{M_{23}(s) \geq n, q_{23}(s)=0\right\} e^{-\left(\mu_{1}-\lambda_{1}\right) s} d s \\
\geq & \log \left\{\frac{\lambda_{2}+\lambda_{3}}{\lambda_{2}+\lambda_{3}+\mu_{1}-\lambda_{1}}\right\}
\end{aligned}
$$

Similarly, since,

$$
\mu_{2}-\lambda_{2}+\mu_{3}-\lambda_{3} \geq \mu_{1}-\lambda_{1}+\mu_{2}-\lambda_{2}
$$

the second term $K_{2} \int_{0}^{\infty} \mathbb{P}\left\{M_{23}(s) \geq n, q_{23}(s)=0\right\} e^{-\left(\mu_{1}-\lambda_{1}+\mu_{2}-\lambda_{2}\right) s} d s$ gives,

$$
\begin{aligned}
& \lim _{n \rightarrow \infty} \frac{1}{n} \log \int_{0}^{\infty} \mathbb{P}\left\{M_{23}(s) \geq n, q_{23}(s)=0\right\} e^{-\left(\mu_{1}-\lambda_{1}+\mu_{2}-\lambda_{2}\right) s} d s \\
\geq & \log \left\{\frac{\lambda_{2}+\lambda_{3}}{\lambda_{2}+\lambda_{3}+\mu_{1}-\lambda_{1}+\mu_{2}-\lambda_{2}}\right\}
\end{aligned}
$$


For the third term $K_{3} \int_{0}^{\infty} \mathbb{P}\left\{M_{23}(s) \geq n, q_{23}(s)=0\right\} e^{-\left(\mu_{1}-\lambda_{1}+\mu_{3}-\lambda_{3}\right) s} d s$, since

$$
\mu_{2}-\lambda_{2}+\mu_{3}-\lambda_{3} \geq \mu_{1}-\lambda_{1}+\mu_{3}-\lambda_{3}
$$

it yields,

$$
\begin{aligned}
& \lim _{n \rightarrow \infty} \frac{1}{n} \log \int_{0}^{\infty} \mathbb{P}\left\{M_{23}(s) \geq n, q_{23}(s)=0\right\} e^{-\left(\mu_{1}-\lambda_{1}+\mu_{3}-\lambda_{3}\right) s} d s \\
\geq & \log \left\{\frac{\lambda_{2}+\lambda_{3}}{\lambda_{2}+\lambda_{3}+\mu_{1}-\lambda_{1}+\mu_{3}-\lambda_{3}}\right\}
\end{aligned}
$$

For the fourth term

$K_{4} \int_{0}^{\infty} \mathbb{P}\left\{M_{23}(s) \geq n, q_{23}(s)=0\right\} e^{-\left(\mu_{1}-\lambda_{1}+\mu_{2}-\lambda_{2}+\mu_{3}-\lambda_{3}\right) s} d s$, since,

$$
\mu_{2}-\lambda_{2}+\mu_{3}-\lambda_{3}<\mu_{1}-\lambda_{1}+\mu_{2}-\lambda_{2}+\mu_{3}-\lambda_{3}
$$

it gives,

$$
\begin{aligned}
& \lim _{n \rightarrow \infty} \frac{1}{n} \log \int_{0}^{\infty} \mathbb{P}\left\{M_{23}(s) \geq n, q_{23}(s)=0\right\} e^{-\left(\mu_{1}-\lambda_{1}+\mu_{2}-\lambda_{2}+\mu_{3}-\lambda_{3}\right) s} d s \\
\geq & \log \left\{\frac{4\left(\lambda_{2}+\lambda_{3}\right)\left(\mu_{2}+\mu_{3}\right)}{\left(\lambda_{2}+\lambda_{3}+\mu_{2}+\mu_{3}+\mu_{1}-\lambda_{1}+\mu_{2}-\lambda_{2}+\mu_{3}-\lambda_{3}\right)^{2}}\right\} \\
= & \log \left\{\frac{4\left(\lambda_{2}+\lambda_{3}\right)\left(\mu_{2}+\mu_{3}\right)}{\left(2 \mu_{2}+2 \mu_{3}+\mu_{1}-\lambda_{1}\right)^{2}}\right\}
\end{aligned}
$$


$\left(\left\llcorner\mathcal{G}^{\circ} \mathcal{E}\right)\right.$

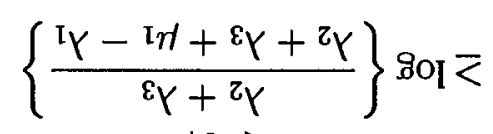

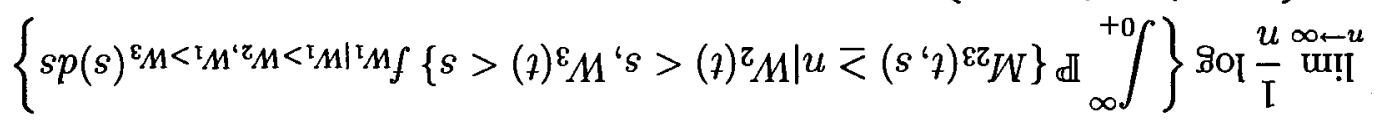

'әдојәдәчL

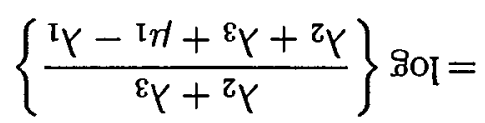

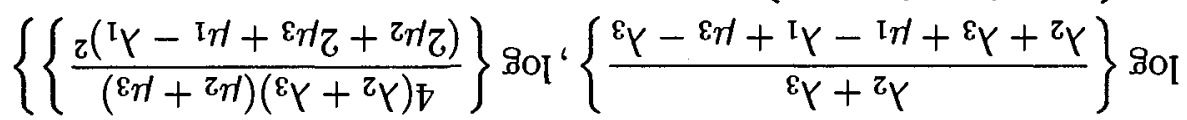

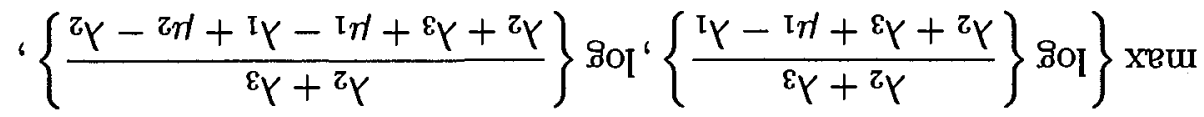

'นә૫工

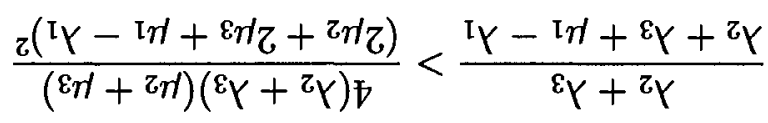

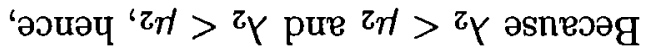

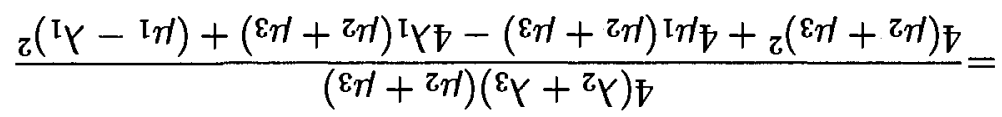

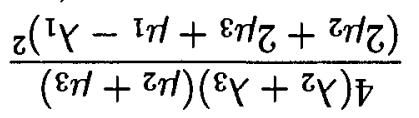

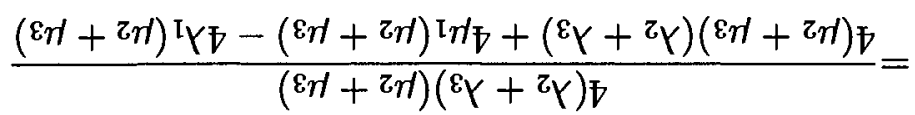

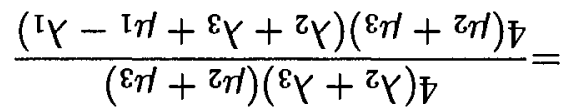

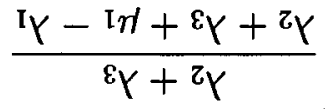


Using similar arguments and by Theorem (3.1), we get when $\mu_{1}-\lambda_{1} \leq \mu_{2}-\lambda_{2} \leq$ $\mu_{3}-\lambda_{3}$ and $\mu_{1}-\lambda_{1}+\mu_{2}-\lambda_{2} \leq \mu_{3}-\lambda_{3}$

$$
\begin{aligned}
& \lim _{n \rightarrow \infty} \frac{1}{n} \log \left\{\int_{0+}^{\infty} \mathbb{P}\left\{M_{23}(t, s) \geq n\right\} f_{W_{1} \mid W_{1}>W_{2}, W_{1}>W_{3}}(s) d s\right\} \\
\leq & \log \left\{\frac{\lambda_{2}+\lambda_{3}}{\lambda_{2}+\lambda_{3}+\mu_{1}-\lambda_{1}}\right\}
\end{aligned}
$$

It is an upper bound of $\lim _{n \rightarrow \infty} \frac{1}{n} \log \left\{\int_{0+}^{\infty} \mathbb{P}\left\{M_{23}(t, s) \geq n \mid W_{2}(t)<s, W_{3}(t)<s\right\}\right.$ - $\left.f_{W_{1} \mid W_{1}>W_{2}, W_{1}>W_{3}}(s) d s\right\}$. The reason is,

$$
\begin{aligned}
& \mathbb{P}\left\{M_{23}(t, s) \geq n\right\} \\
= & \mathbb{P}\left\{M_{23}(t, s) \geq n \mid W_{2}(t)<s, W_{3}(t)<s\right\} \cdot \mathbb{P}\left\{W_{2}(t)<s, W_{3}(t)<s\right\} \\
+ & \mathbb{P}\left\{M_{23}(t, s) \geq n \mid W_{2}(t) \geq s, W_{3}(t) \geq s\right\} \cdot \mathbb{P}\left\{W_{2}(t) \geq s, W_{3}(t) \geq s\right\} \\
\geq & \mathbb{P}\left\{M_{23}(t, s) \geq n \mid W_{2}(t)<s, W_{3}(t)<s\right\} \cdot \mathbb{P}\left\{W_{2}(t)<s, W_{3}(t)<s\right\} \\
+ & \mathbb{P}\left\{M_{23}(t, s) \geq n \mid W_{2}(t)<s, W_{3}(t)<s\right\} \cdot \mathbb{P}\left\{W_{2}(t) \geq s, W_{3}(t) \geq s\right\} \\
= & \mathbb{P}\left\{M_{23}(t, s) \geq n \mid W_{2}(t)<s, W_{3}(t)<s\right\}
\end{aligned}
$$

Since the upper bound and the lower bound agree with each other, by the Squeezing theorem and (3.12), we get

$$
\begin{aligned}
& \lim _{n \rightarrow \infty} \frac{1}{n} \log \left\{\mathbb{P}\left\{M_{23}\left(t, W_{*}(t)\right) \geq n \mid W_{1}(t)>W_{2}(t), W_{1}(t)>W_{3}(t)\right\}\right\} \\
= & \log \left\{\frac{\lambda_{2}+\lambda_{3}}{\lambda_{2}+\lambda_{3}+\mu_{1}-\lambda_{1}}\right\}
\end{aligned}
$$

To determine $\mathbb{P}\left\{q^{r}(t) \geq n\right\}$ for large $n$, we also need to compute $\mathbb{P}\left\{M_{13}\left(t, W_{*}(t)\right) \geq\right.$ $\left.n \mid W_{2}(t)>W_{1}(t), W_{2}(t)>W_{3}(t)\right\}$ and $\mathbb{P}\left\{M_{12}\left(t, W_{*}(t)\right) \geq n \mid W_{3}(t)>W_{1}(t), W_{3}(t)>\right.$ 
$\left.W_{2}(t)\right\}$ (See (3.11)). Using similar arguments above, we have, when $\mu_{1}-\lambda_{1} \leq$ $\mu_{2}-\lambda_{2} \leq \mu_{3}-\lambda_{3}$ and $\mu_{1}-\lambda_{1}+\mu_{2}-\lambda_{2} \leq \mu_{3}-\lambda_{3}$,

$$
\begin{aligned}
& \lim _{n \rightarrow \infty} \frac{1}{n} \log \left\{\mathbb{P}\left\{M_{13}\left(t, W_{*}(t)\right) \geq n \mid W_{2}(t)>W_{1}(t), W_{2}(t)>W_{3}(t)\right\}\right\} \\
= & \max \left\{\log \left\{\frac{\lambda_{1}+\lambda_{3}}{\lambda_{1}+\lambda_{3}+\mu_{2}-\lambda_{2}}\right\}, \log \left\{\frac{4\left(\lambda_{1}+\lambda_{3}\right)\left(\mu_{1}+\mu_{3}\right)}{\left(\lambda_{1}+\mu_{1}+2 \mu_{3}+\mu_{2}-\lambda_{2}\right)^{2}}\right\}\right\}
\end{aligned}
$$

$$
\begin{aligned}
& \lim _{n \rightarrow \infty} \frac{1}{n} \log \left\{\mathbb{P}\left\{M_{12}\left(t, W_{*}(t)\right) \geq n \mid W_{3}(t)>W_{1}(t), W_{3}(t)>W_{2}(t)\right\}\right\} \\
= & \log \left\{\frac{4\left(\lambda_{1}+\lambda_{2}\right)\left(\mu_{1}+\mu_{2}\right)}{\left(\lambda_{1}+\lambda_{2}+\mu_{1}+\mu_{2}+\mu_{3}-\lambda_{3}\right)^{2}}\right\}
\end{aligned}
$$

When $\mu_{1}-\lambda_{1} \leq \mu_{2}-\lambda_{2} \leq \mu_{3}-\lambda_{3}$ and $\mu_{1}-\lambda_{1}+\mu_{2}-\lambda_{2} \leq \mu_{3}-\lambda_{3}$, combining (3.60), (3.61), (3.62) and (3.11), we get Theorem 3.2. 


\section{Chapter 4}

\section{$m-M / M / 1$ Case}

In the previous chapter we have discussed the case that there are three $M / M / 1$ queues in the DN system. In this chapter, we extend the number of queues from three to any positive integer $m \geq 2$. The analysis of the conclusion is far more complicated than that for the $3-M / M / 1$ case, when the number of queues goes large. Therefore, we make the assumptions that all $m M / M / 1$ queues having the same arriving and service rates.

\subsection{Model Description}

The model is shown in Figure 4.1, and it is similar to the one discussed in the previous chapter. In the DN system, there are $m$ parallel $M / M / 1$ queues. Each customer enters queue $i$ with probability with $p_{i}=\frac{1}{m}$. Hence the arrival rates for each queue $\lambda_{i}=\frac{\lambda}{m}$ are also equal. We also assume that $m$ servers in the DN have the same service rates, or $\mu_{1}=\mu_{2}=\cdots=\mu_{m}$. Therefore, in the DN system, we have $m$ parallel identical queues. For the stability reason, we still assume $\lambda_{i}<\mu_{i}$ 


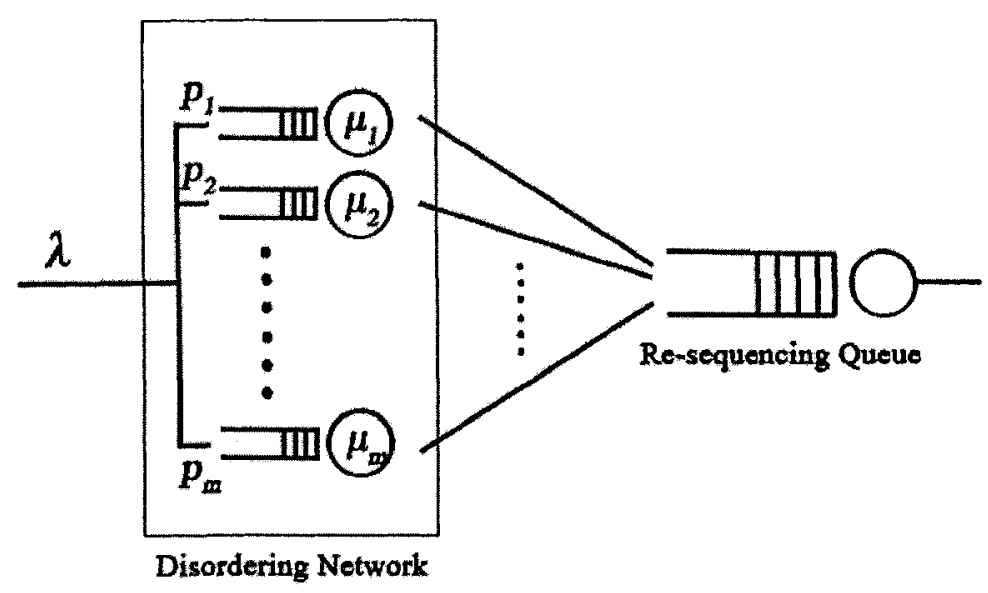

Figure 4.1: $m-M / M / 1$ Network and Re-sequencing Model

for all $i=1,2, \cdots, m$, or $\lambda<m \mu_{1}$.

The RSQ is defined in the same way as in previous chapter, that is, if the customers are properly ordered in RSQ, then they are immediately transmitted, otherwise they will wait in the RSQ.

As we denoted before, $q^{r}$ is the stationary size of the RSQ, and we also discuss the large deviation type of result about $q^{r}$.

\subsubsection{Setup}

All the definitions of $V(t), V^{c}(t), C_{*}(t), W_{*}(t), I_{*}(t)$ and $E(t, s, n)$ are the same as before. At time $t$, the oldest customer $C_{*}(t)$ is being served in queue $I_{*}(t)$. The 
size of RSQ is greater or equal to $n$ at time $t$ means that at least $n$ customers who arrived after $C_{*}(t)$ but left the DN by time $t$. Using the same method, we can get the same conclusion that

$$
\mathbb{P}\left\{q^{r}(t) \geq n\right\}=\mathbb{P}\left\{E\left(t, W_{*}(t), n\right)\right\}
$$

\subsubsection{The Relationship between $\mathbb{P}\left\{q^{r}(t) \geq n\right\}$ and $M_{(i)}(t, s)$}

Let $M_{(i)}(t, s)$ be the total number of customers who arrived at the DN system, but not entering queue $i$, during the time interval $(t-s, t]$ and departed by time $t$, where $i=1,2, \cdots, m . W_{i}(t)$ is still defined as the duration in which the customer in service at queue $i$ has stayed in the queue.

At time $t$, if the oldest customer $C_{*}(t)$ is being served in queue $i$, then the event $\left\{q^{r}(t) \geq n\right\}$ is equivalent to the event $\left\{M_{(i)}\left(t, W_{i}(t)\right) \geq n\right\}$. According to earlier discussions, the relationship between $\mathbb{P}\left\{q^{r}(t) \geq n\right\}$ and $M_{(i)}(t, s)$ can be expressed as follows,

$$
\begin{aligned}
& \mathbb{P}\left\{q^{r}(t) \geq n\right\} \\
= & \mathbb{P}\left\{E\left(t, W_{*}(t), n\right)\right\} \\
= & \sum_{i=1}^{m} \mathbb{P}\left\{M_{(i)}\left(t, W_{*}(t)\right) \geq n \mid \bigcap_{\substack{j=1 \\
j \neq i}}^{m} W_{i}(t)>W_{j}(t)\right\} \mathbb{P}\left\{\bigcap_{\substack{j=1 \\
j \neq i}}^{m} W_{i}(t)>W_{j}(t)\right\}
\end{aligned}
$$




\subsubsection{Bounds}

From the analysis of 4.1 .2 , we have obtained that the $\mathbb{P}\left\{q^{r}(t) \geq n\right\}$ consists of $m$ symmetric parts: $\mathbb{P}\left\{M_{(i)}\left(t, W_{*}(t)\right) \geq n \mid \bigcap_{\substack{j=1 \\ j \neq i}}^{m} W_{i}(t)>W_{j}(t)\right\}, i$ is from 1 to $m$. Without losing generality, in this section, we just discuss the component $\mathbb{P}\left\{M_{(1)}\left(t, W_{*}(t)\right) \geq n \mid \bigcap_{j=2}^{m} W_{1}(t)>W_{j}(t)\right\}$. At this situation, $W_{*}(t)=W_{1}(t)$.

For $n>0$, still conditioning on $W_{1}(t)$, we have,

$$
\begin{aligned}
& \mathbb{P}\left\{M_{(1)}\left(t, W_{*}(t)\right) \geq n \mid \bigcap_{j=2}^{m} W_{1}(t)>W_{j}(t)\right\} \\
= & \int_{0+}^{\infty} \mathbb{P}\left\{M_{(1)}\left(t, W_{*}(t)\right) \geq n \mid W_{1}(t)=s, \bigcap_{j=2}^{m} W_{1}(t)>W_{j}(t)\right\} \\
& \cdot f_{W_{1} \mid \bigcap_{j=2}^{m} W_{1}>W_{j}}(s) d s \\
= & \int_{0+}^{\infty} \mathbb{P}\left\{M_{(1)}(t, s) \geq n \mid \bigcap_{j=2}^{m} W_{j}(t)<s\right\} f_{W_{1} \mid \bigcap_{j=2}^{m} W_{1}>W_{j}}(s) d s
\end{aligned}
$$

where $f_{W_{1} \mid \bigcap_{j=2}^{m} W_{1}>W_{j}}(s)$ denotes the condition density of $W_{1}(t)$ given $\left\{\bigcap_{j=2}^{m} W_{1}(t)>\right.$ $\left.W_{j}(t)\right\}$ and the second quality is true because all these $m$ queues in DN system are independent.

Now, we need to find the expression for $f_{W_{1} \mid \bigcap_{j=2}^{m} W_{1}>W_{j}}(s)$. For $x \geq 0$, condition on $W_{1}(t)$. According to (3.13), it is easy to check the following result. 
If $m$ is odd,

$$
\begin{aligned}
& \mathbb{P}\left\{W_{1}(t)>x, \bigcap_{j=2}^{m} W_{1}(t)>W_{j}(t)\right\} \\
= & \rho_{1} e^{-\left(\mu_{1}-\lambda_{1}\right) x}-\sum_{k=2}^{m} \rho_{1} \rho_{k} \frac{\mu_{1}-\lambda_{1}}{\mu_{1}-\lambda_{1}+\mu_{k}-\lambda_{k}} e^{-\left(\mu_{1}-\lambda_{1}+\mu_{k}-\lambda_{k}\right) x} \\
& +\sum_{p=2}^{m} \sum_{q=p+1}^{m} \rho_{1} \rho_{p} \rho_{q} \frac{\mu_{1}-\lambda_{1}}{\mu_{1}-\lambda_{1}+\mu_{p}-\lambda_{p}+\mu_{q}-\lambda_{q}} e^{-\left(\mu_{1}-\lambda_{1}+\mu_{p}-\lambda_{p}+\mu_{q}-\lambda_{q}\right) x} \\
& +\cdots \\
& +\rho_{1} \rho_{2} \cdots \rho_{m} \frac{\mu_{1}-\lambda_{1}}{\mu_{1}-\lambda_{1}+\mu_{2}-\lambda_{2}+\cdots+\mu_{m}-\lambda_{m}} e^{-\left(\mu_{1}-\lambda_{1}+\mu_{2}-\lambda_{2}+\cdots+\mu_{m}-\lambda_{m}\right) x}
\end{aligned}
$$

If $m$ is even,

$$
\begin{aligned}
& \mathbb{P}\left\{W_{1}(t)>x, \bigcap_{j=2}^{m} W_{1}(t)>W_{j}(t)\right\} \\
= & \rho_{1} e^{-\left(\mu_{1}-\lambda_{1}\right) x}-\sum_{k=2}^{m} \rho_{1} \rho_{k} \frac{\mu_{1}-\lambda_{1}}{\mu_{1}-\lambda_{1}+\mu_{k}-\lambda_{k}} e^{-\left(\mu_{1}-\lambda_{1}+\mu_{k}-\lambda_{k}\right) x} \\
& +\sum_{p=2}^{m} \sum_{q=p+1}^{m} \rho_{1} \rho_{p} \rho_{q} \frac{\mu_{1}-\lambda_{1}}{\mu_{1}-\lambda_{1}+\mu_{p}-\lambda_{p}+\mu_{q}-\lambda_{q}} e^{-\left(\mu_{1}-\lambda_{1}+\mu_{p}-\lambda_{p}+\mu_{q}-\lambda_{q}\right) x} \\
& +\cdots \\
& -\rho_{1} \rho_{2} \cdots \rho_{m} \frac{\mu_{1}-\lambda_{1}}{\mu_{1}-\lambda_{1}+\mu_{2}-\lambda_{2}+\cdots+\mu_{m}-\lambda_{m}} e^{-\left(\mu_{1}-\lambda_{1}+\mu_{2}-\lambda_{2}+\cdots+\mu_{m}-\lambda_{m}\right) x}
\end{aligned}
$$

There are totally $2^{m-1}$ items in (4.5).

Similarly, based on the method of 3.14 , it gives that 
when $m$ is odd,

$$
\begin{aligned}
& \mathbb{P}\left\{\bigcap_{j=2}^{m} W_{1}(t)>W_{j}(t)\right\} \\
= & \rho_{1}-\sum_{k=2}^{m} \rho_{1} \rho_{k} \frac{\mu_{1}-\lambda_{1}}{\mu_{1}-\lambda_{1}+\mu_{k}-\lambda_{k}} \\
& +\sum_{p=2}^{m} \sum_{q=p+1}^{m} \rho_{1} \rho_{p} \rho_{q} \frac{\mu_{1}-\lambda_{1}}{\mu_{1}-\lambda_{1}+\mu_{p}-\lambda_{p}+\mu_{q}-\lambda_{q}} \\
& +\cdots \\
& +\rho_{1} \rho_{2} \cdots \rho_{m} \frac{\mu_{1}-\lambda_{1}}{\mu_{1}-\lambda_{1}+\mu_{2}-\lambda_{2}+\cdots+\mu_{m}-\lambda_{m}}
\end{aligned}
$$

when $m$ is even,

$$
\begin{aligned}
& \mathbb{P}\left\{\bigcap_{j=2}^{m} W_{1}(t)>W_{j}(t)\right\} \\
&= \rho_{1}-\sum_{k=2}^{m} \rho_{1} \rho_{k} \frac{\mu_{1}-\lambda_{1}}{\mu_{1}-\lambda_{1}+\mu_{k}-\lambda_{k}} \\
&+\sum_{p=2}^{m} \sum_{q=p+1}^{m} \rho_{1} \rho_{p} \rho_{q} \frac{\mu_{1}-\lambda_{1}}{\mu_{1}-\lambda_{1}+\mu_{p}-\lambda_{p}+\mu_{q}-\lambda_{q}} \\
&+\ldots \\
&-\rho_{1} \rho_{2} \cdots \rho_{m} \frac{\mu_{1}-\lambda_{1}}{\mu_{1}-\lambda_{1}+\mu_{2}-\lambda_{2}+\cdots+\mu_{m}-\lambda_{m}} \\
& \text { Since } \bar{F}_{W_{1} \mid \bigcap_{j=2}^{m} W_{1}>W_{j}}(x)=\frac{\mathbb{P}\left\{W_{1}(t)>x, \bigcap_{j=2}^{m} W_{1}(t)>W_{j}(t)\right\}}{\mathbb{P}\left\{\bigcap_{j=2}^{m} W_{1}(t)>W_{j}(t)\right\}}
\end{aligned}
$$

and $f_{W_{1} \mid \bigcap_{j=2}^{m} W_{1}>W_{j}}(x)=\frac{d}{d x}\left(1-\bar{F}_{W_{1} \mid \bigcap_{j=2}^{m} W_{1}>W_{j}}(x)\right)$, we can have that, 
when $m$ is odd,

$$
\begin{aligned}
& f_{W_{1} \mid \bigcap_{j=2}^{m} W_{1}>W_{j}}(x) \\
& =A_{1} e^{-\left(\mu_{1}-\lambda_{1}\right) x}-\sum_{k=2}^{m} A_{k} e^{-\left(\mu_{1}-\lambda_{1}+\mu_{k}-\lambda_{k}\right) x} \\
& \quad+\sum_{p=2}^{m} \sum_{q=p+1}^{m} A_{p q} e^{-\left(\mu_{1}-\lambda_{1}+\mu_{p}-\lambda_{p}+\mu_{q}-\lambda_{q}\right) x} \\
& \quad+\cdots \\
& \quad+A_{12 \cdots m} e^{-\left(\mu_{1}-\lambda_{1}+\mu_{2}-\lambda_{2}+\cdots+\mu_{m}-\lambda_{m}\right) x}
\end{aligned}
$$

where $A_{1}, A_{2}, \cdots, A_{m}, A_{23}, \cdots, A_{12 \cdots m}$ are $2^{m-1}$ positive constants; when $m$ is even,

$$
\begin{aligned}
& f_{W_{1} \mid \cap_{j=2}^{m} W_{1}>W_{j}}(x) \\
& =B_{1} e^{-\left(\mu_{1}-\lambda_{1}\right) x}-\sum_{k=2}^{m} B_{k} e^{-\left(\mu_{1}-\lambda_{1}+\mu_{k}-\lambda_{k}\right) x} \\
& \quad+\sum_{p=2}^{m} \sum_{q=p+1}^{m} B_{p q} e^{-\left(\mu_{1}-\lambda_{1}+\mu_{p}-\lambda_{p}+\mu_{q}-\lambda_{q}\right) x} \\
& +\cdots \\
& \quad-B_{12 \cdots m} e^{-\left(\mu_{1}-\lambda_{1}+\mu_{2}-\lambda_{2}+\cdots+\mu_{m}-\lambda_{m}\right) x}
\end{aligned}
$$

where $B_{1}, B_{2}, \cdots, B_{m}, B_{23}, \cdots, B_{12 \cdots m}$ are $2^{m-1}$ positive constants. 
For a lower bound, by the definition of conditional probability,

$$
\begin{aligned}
& \int_{0+}^{\infty} \mathbb{P}\left\{M_{(1)}(t, s) \geq n \mid \bigcap_{j=2}^{m} W_{j}(t)<s\right\} f_{W_{1} \mid \cap_{j=2}^{m} W_{1}>W_{j}}(s) d s \\
= & \int_{0+}^{\infty} \mathbb{P}\left\{M_{(1)}(t, s) \geq n, \bigcap_{j=2}^{m} W_{j}(t)<s\right\} \frac{f_{W_{1} \mid \bigcap_{j=2}^{m} W_{1}>W_{j}}(s)}{\mathbb{P}\left\{\bigcap_{j=2}^{m} W_{j}(t)<s\right\}} d s \\
\geq & \int_{0+}^{\infty} \mathbb{P}\left\{M_{(1)}(t, s) \geq n, \bigcap_{j=2}^{m} W_{j}(t)=0\right\} f_{W_{1} \mid \bigcap_{j=2}^{m} W_{1}>W_{j}}(s) d s \\
= & \int_{0+}^{\infty} \mathbb{P}\left\{M_{(1)}(t, s) \geq n, \bigcap_{j=2}^{m} q_{j}(t)=0\right\} f_{W_{1} \mid \bigcap_{j=2}^{m} W_{1}>W_{j}}(s) d s
\end{aligned}
$$

In above inequality, $q_{i}(t)$ has the same definition as before, and from the definition of $W_{i}(t)$, we can obtain that the event $\left\{W_{i}(t)=0\right\}$ is equivalent to the event $\left\{q_{i}(t)=0\right\}$.

For a upper bound,

$$
\begin{aligned}
& \int_{0+}^{\infty} \mathbb{P}\left\{M_{(1)}(t, s) \geq n \mid \bigcap_{j=2}^{m} W_{j}(t)<s\right\} f_{W_{1} \mid \bigcap_{j=2}^{m} W_{1}>W_{j}}(s) d s \\
= & \int_{0+}^{\infty} \mathbb{P}\left\{M_{(1)}(t, s) \geq n, \bigcap_{j=2}^{m} W_{j}(t)<s\right\} \frac{f_{W_{1} \mid \bigcap_{j=2}^{m} W_{1}>W_{j}}(s)}{\mathbb{P}\left\{\bigcap_{j=2}^{m} W_{j}(t)<s\right\}} d s \\
\leq & \int_{0+}^{\infty} \mathbb{P}\left\{M_{(1)}(t, s) \geq n\right\} \frac{f_{W_{1} \mid \bigcap_{j=2}^{m} W_{1}>W_{j}}(s)}{\mathbb{P}\left\{\bigcap_{j=2}^{m} W_{j}(t)=0\right\}} d s \\
= & \frac{1}{\left(1-\rho_{1}\right)^{m-1}} \int_{0+}^{\infty} \mathbb{P}\left\{M_{(1)}(t, s) \geq n\right\} f_{W_{1} \mid \bigcap_{j=2}^{m} W_{1}>W_{j}}(s) d s
\end{aligned}
$$

In the last equation, we use the fact that all queue $i$ are independent and $\mathbb{P}\left\{W_{i}(t)=\right.$ $0\}=1-\rho_{i}$. We also use the assumption in this chapter that $\lambda_{1}=\lambda_{2}=\cdots \lambda_{m}$ and $\mu_{1}=\mu_{2}=\cdots=\mu_{m}$. 


\subsection{Computation of $\mathbb{P}\{\hat{M}(\hat{T}) \geq n\}$}

The discussion presented here is similar to that for the computation of $\mathbb{P}\{M(T) \geq$ $n$ \} in Section 3.2, except that the number of queues increases from two to $m-1$ and use the similar method to find $\mathbb{P}\{\hat{M}(\hat{T}) \geq n\}$. We consider $m-1$ independent and stationary $M / M / 1$ queues with arrival rates $\lambda_{i}$ and departure rates $\mu_{i}$, where $i$ is from 1 to $m-1$, respectively. Suppose that the capacity of each queue is infinity. We assume $\lambda_{i}<\mu_{i}$ for all $i$, so that all queues are stable.

$\hat{M}(t)$ is defined as the total number of those customers, who arrived at one of these $m-1$ queues during interval $[0, t]$ and departed by time $t$. Let $\hat{T}$ be an exponential random variable with mean $\frac{1}{\mu_{m}-\lambda_{m}}$ and $\hat{T}$ is independent of these $m-1$ queueing processes. The density function of $\hat{T}$ is $f_{\hat{T}}(t)=\left(\mu_{m}-\lambda_{m}\right) e^{-\left(\mu_{m}-\lambda_{m}\right) t}$, for $t \geq 0$.

\subsubsection{Case 1: $\sum_{i=1}^{m-1}\left(\mu_{i}-\lambda_{i}\right) \geq \mu_{m}-\lambda_{m}$}

\section{The Upper Bound}

According to the analysis in Section 3.2 .1 , we define a new variable $\hat{M}^{*}(t)$, which is the total number of those customers, who arrived at either of these $m-1$ queues during interval $[0, t]$. It is obvious that

$$
\hat{M}(t) \leq \hat{M}^{*}(t)
$$


In fact, $\hat{M}^{*}(t)$ is the total number of arrivals from $m-1$ independent Poisson processes. Therefore, by the property of independent Poisson process and using the same method as 3.18 , we can get,

$$
\begin{aligned}
& \mathbb{P}\{\hat{M}(\hat{T}) \geq n\} \\
\leq & \left(\frac{\sum_{i=1}^{m-1} \lambda_{i}}{\sum_{i=1}^{m-1} \lambda_{i}+\mu_{m}-\lambda_{m}}\right)^{n}
\end{aligned}
$$

\section{The Lower Bound}

The total size of the $m-1$ independent queues at time $t$ is denoted by $\hat{q}(t)$. And let $\hat{D}(t)$ be the total number of departures during the interval $[0, t]$. The departure process of each queue is independent Poisson with rate $\lambda_{i}$, so by the Poisson property $\hat{D}(t)$ is Poisson distributed with $\sum_{i=1}^{m-1} \lambda_{i}$. Similarly, we also define $\tilde{\hat{q}}(t)$ be the queue size at time $t$ of $M / M / 1$ queue having arrival rate $\sum_{i=1}^{m-1} \lambda_{i}$ and departure rate $\sum_{i=1}^{m-1} \mu_{i}$ and $\tilde{\hat{D}}(t)$ is the number of departure during $[0, t]$ of this queue. Then $\tilde{\hat{D}}(t)$ has the same distribution as $\hat{D}(t)$. Hence, by conditioning on $\hat{q}(t)$ we obtain,

$$
\mathbb{P}\{\hat{M}(t)=k\} \geq C \cdot \mathbb{P}\{\hat{\hat{D}}(t)=k \bigcap \tilde{\hat{q}}(0)=0\}
$$

The reason is according to (3.19) and Remark 3.2.

Replace $\mathbb{P}\{\tilde{\hat{D}}(t)=k \cap \tilde{\hat{q}}(0)=0\}$ in 4.13 by the result of ([7]) (page 199), and 
simplified as (3.20) and (3.21), we get,

$$
\begin{aligned}
& \mathbb{P}\{\hat{M}(t)=k\} \\
\geq & C(1-\hat{\rho}) \frac{1}{k+1} \frac{\left[\left(\sum_{i=1}^{m-1} \lambda_{i}\right) t\right]^{k} e^{-\left(\sum_{i=1}^{m-1} \lambda_{i}\right) t}}{k !} \mathbb{P}\left\{Y_{\left(\sum_{j=1}^{m-1} \mu_{j}\right) t} \geq k\right\}
\end{aligned}
$$

where $\hat{\rho}=\frac{\sum_{i=1}^{m-1} \lambda_{i}}{\sum_{j=1}^{m-1} \mu_{j}}$ and $Y_{\left(\sum_{j=1}^{m-1} \mu_{j}\right) t}$ is a Poisson random variable with mean $\left(\sum_{j=1}^{m-1} \mu_{j}\right) t$

Based on Remark 3.3, we choose $\hat{t}_{0}=\frac{n}{\sum_{i=1}^{m-1} \lambda_{i}+\mu_{m}-\lambda_{m}}$, then

$$
\begin{aligned}
\mathbb{P}\{\hat{M}(\hat{T}) \geq n\} & \geq \mathbb{P}\left\{\hat{M}(\hat{T}) \geq n \bigcap \hat{T} \geq t_{0}\right\} \\
& \geq \mathbb{P}\left\{\hat{M}\left(t_{0}\right) \geq n \bigcap \hat{T} \geq t_{0}\right\} \\
& =\mathbb{P}\left\{\hat{M}\left(t_{0}\right) \geq n\right\} \mathbb{P}\left\{\hat{T} \geq t_{0}\right\} \\
& =\sum_{k=n}^{\infty} \mathbb{P}\left\{\hat{M}\left(t_{0}\right)=k\right\} \mathbb{P}\left\{\hat{T} \geq t_{0}\right\}
\end{aligned}
$$

Combining (4.14) and (4.15), yields

$$
\begin{aligned}
& \mathbb{P}\{\hat{M}(\hat{T}) \geq n\} \\
\geq & C(1-\hat{\rho}) \sum_{k=n}^{\infty} \frac{1}{k+1} \frac{\left[\left(\sum_{i=1}^{m-1} \lambda_{i}\right) t_{0}\right]^{k} e^{-\left(\sum_{i=1}^{m-1} \lambda_{i}\right) t_{0}}}{k !} \mathbb{P}\left\{Y_{\left(\sum_{j=1}^{m-1} \mu_{j}\right) t_{0}} \geq k\right\} \cdot e^{-\left(\mu_{m}-\lambda_{m}\right) t_{0}} \\
\geq & C(1-\hat{\rho}) \frac{1}{n+1} \frac{\left[\left(\sum_{i=1}^{m-1} \lambda_{i}\right) t_{0}\right]^{n} e^{-\left(\sum_{i=1}^{m-1} \lambda_{i}\right) t_{0}}}{n !} \mathbb{P}\left\{Y_{\left(\sum_{j=1}^{m-1} \mu_{j}\right) t_{0}} \geq n\right\} \cdot e^{-\left(\mu_{m}-\lambda_{m}\right) t_{0}}
\end{aligned}
$$

By the similar method, we can prove that for any $\epsilon \geq 0$, there exists some integer 
$N \geq 0$ such that for all $n \geq N$

$$
\mathbb{P}\left\{Y_{\left(\sum_{i=1}^{m-1} \mu_{i}\right) t_{0}} \geq n\right\} \geq \frac{1}{2}-\epsilon
$$

From the Stirling's approximation, we replace $n$ ! by $\sqrt{2 \pi n} n^{n} e^{-n}$. Thus, when $n$ is large enough, the lower bound of $\mathbb{P}\{\hat{M}(\hat{T}) \geq n\}$ can be computed from (4.16)

$$
\begin{aligned}
& \mathbb{P}\{M(\hat{T}) \geq n\} \\
\geq & \frac{1}{4} C(1-\hat{\rho})(1-2 \epsilon) \frac{1}{n+1} \frac{\left(\frac{\sum_{i=1}^{m-1} \lambda_{i}}{\sum_{i=1}^{m-1} \lambda_{i}+\mu_{m}-\lambda_{m}}\right)^{n} n^{n} \exp \left\{-\frac{\sum_{i=1}^{m-1} \lambda_{i}}{\sum_{i=1}^{m-1} \lambda_{i}+\mu_{m}-\lambda_{m}} n\right\}}{\sqrt{2 \pi n} n^{n} e^{-n}} \\
& \cdot \exp \left\{-\frac{\mu_{m}-\lambda_{m}}{\sum_{i=1}^{m-1} \lambda_{i}+\mu_{m}-\lambda_{m}} n\right\} \\
= & \frac{C(1-\hat{\rho})(1-2 \epsilon)}{4 \sqrt{2 \pi n}(n+1)}\left(\frac{\sum_{i=1}^{m-1} \lambda_{i}}{\sum_{i=1}^{m-1} \lambda_{i}+\mu_{m}-\lambda_{m}}\right)^{n}
\end{aligned}
$$

\section{Large Deviation Result for $\hat{M}(\hat{T})$ in Case 1}

We have found both lower and upper bounds when $\sum_{i=1}^{m-1}\left(\mu_{i}-\lambda_{i}\right) \geq \mu_{m}-\lambda_{m}$, and it is easy to show that if we take the logarithm and multiply $\frac{1}{n}$ on both bounds, the limit of them will converge to the same value, that is, $\log \left\{\frac{\sum_{i=1}^{m-1} \lambda_{i}}{\sum_{i=1}^{m-1} \lambda_{i}+\mu_{m}-\lambda_{m}}\right\}$.

By the Squeezing Theorem,

$$
\lim _{n \rightarrow \infty} \frac{1}{n} \log \mathbb{P}\{\hat{M}(\hat{T}) \geq n\}=\log \left\{\frac{\sum_{i=1}^{m-1} \lambda_{i}}{\sum_{i=1}^{m-1} \lambda_{i}+\mu_{m}-\lambda_{m}}\right\}
$$




\subsubsection{Case 2: $\sum_{i=1}^{m-1}\left(\mu_{i}-\lambda_{i}\right)<\mu_{m}-\lambda_{m}$}

\section{The Lower Bound}

Conditioning on $\hat{T}$ and the inequality (4.16), we have

$$
\begin{aligned}
& \mathbb{P}\{\hat{M}(\hat{T}) \geq n\} \\
= & \int_{0}^{\infty} \sum_{k=n}^{\infty} \mathbb{P}\{\hat{M}(t)=n \mid \hat{T}=t\} f_{\hat{T}}(t) d t \\
\geq & \frac{C(1-\hat{\rho})\left(\mu_{m}-\lambda_{m}\right)}{n+1} \int_{0}^{\infty} \frac{\left[\left(\sum_{i=1}^{m-1} \lambda_{i}\right) t\right]^{n} e^{-\left(\sum_{i=1}^{m-1} \lambda_{i}\right) t}}{n !} \mathbb{P}\left\{Y_{\left(\sum_{j=1}^{m-1} \mu_{j}\right) t}=n\right\} e^{-\left(\mu_{m}-\lambda_{m}\right) t} d t \\
= & C(1-\hat{\rho}) \frac{\mu_{m}-\lambda_{m}}{\sum_{i=1}^{m-1} \lambda_{i}+\sum_{j=1}^{m-1} \mu_{j}+\mu_{m}-\lambda_{m}} \frac{1}{n+1} \frac{(2 n) !}{n ! n !}\left(\sum_{i=1}^{m-1} \lambda_{i}\right)^{n}\left(\sum_{j=1}^{m-1} \mu_{j}\right)^{n} \\
& \cdot \frac{1}{\left(\sum_{i=1}^{m-1} \lambda_{i}+\sum_{j=1}^{m-1} \mu_{j}+\mu_{m}-\lambda_{m}\right)^{2 n}}
\end{aligned}
$$

We do not give the details of the simplification here, because it is very similar to (3.33).

Using the Stirling's approximation, for large enough $n$, we have inequality,

$$
\begin{aligned}
\frac{(2 n) !}{n ! n !} & =\frac{\sqrt{4 \pi n}(2 n)^{2 n} e^{-2 n}(1+O(1 / n))}{\left[\sqrt{2 \pi n}(n)^{n} e^{-n}(1+O(1 / n))\right]^{2}} \\
& \geq \frac{C_{1}}{\sqrt{n}} 4^{n}
\end{aligned}
$$

for some positive constant $C_{1}$. 
Hence, we can get the lower bound for this case,

$$
\begin{aligned}
& \mathbb{P}\{\hat{M}(\hat{T}) \geq n\} \\
\geq & M_{3} \frac{1}{\sqrt{n}(n+1)}\left(\frac{4\left(\sum_{i=1}^{m-1} \lambda_{i}\right)\left(\sum_{j=1}^{m-1} \mu_{j}\right)}{\left(\sum_{i=1}^{m-1} \lambda_{i}+\sum_{j=1}^{m-1} \mu_{j}+\mu_{m}-\lambda_{m}\right)^{2}}\right)^{n}
\end{aligned}
$$

where $M_{3}$ is a positive constant by combining all constant items, which does not dependent on $n$.

\section{The Upper Bound}

To compute the upper bound, we will apply the similar method as in Section 3.2.2. Instead of showing the details in this part, we will just give the way of proof.

Comparing with the upper bound of Section 3.2.1, we also need give a tighter bound in this case.

$$
\mathbb{P}\{\hat{M}(t) \geq n\}
$$

$\leq \mathbb{P}\{$ at least $n$ customers arrived to the $(m-1)-M / M / 1$ system during the interval $[0, t]$, and at least $n$ customers are served in (4.23) the same interval\}

$$
\leq \sum_{k=n}^{\infty} \mathbb{P}\left\{\hat{M}^{*}(t)=k\right\} \mathbb{P}\left\{\sum_{l=1}^{n} \hat{Z}_{l} \leq t\right\}
$$

where

$$
\mathbb{P}\left\{\hat{M}^{*}(t)=k\right\}=\frac{\left[\left(\sum_{i=1}^{m-1} \lambda_{i}\right) t\right]^{k} e^{-\left(\sum_{i=1}^{m-1} \lambda_{i}\right) t}}{k !}
$$


where $\hat{Z}_{1}, \hat{Z}_{2}, \cdots, \hat{Z}_{n}$ are i.i.d. service times of $M / M / 1$ queues with arrival rates $\sum_{i=1}^{m-1} \lambda_{i}$ and service rates $\sum_{j=1}^{m-1} \mu_{j}$. In the last step, we still use the fact that these $M / M / 1$ queue has the same departure distribution with the $(m-1)-M / M / 1$ system. Since the pdf of $\sum_{l=1}^{n} \hat{Z}_{l}$ is

$$
f(t)=\frac{\left(\sum_{i=1}^{m-1} \mu_{i}\right) e^{-\left(\sum_{i=1}^{m-1} \mu_{i}\right) t}\left[\left(\sum_{i=1}^{m-1} \mu_{i}\right) t\right]^{n-1}}{(n-1) !}
$$

Thus, from all the results above, we have that

$$
\begin{aligned}
& \mathbb{P}\{\hat{M}(\hat{T}) \geq n\} \\
= & \int_{0}^{\infty} \sum_{k=n}^{\infty} \mathbb{P}\{\hat{M}(t)=n \mid \hat{T}=t\} f_{\hat{T}}(t) d t \\
\leq & \sum_{k=n}^{\infty} \int_{0}^{\infty} \mathbb{P}\left\{\hat{M}^{*}(t)=k\right\} \mathbb{P}\left\{\sum_{l=1}^{n} \hat{Z}_{l} \leq t\right\} f_{T}(t) d t \\
= & \sum_{k=n}^{\infty} \int_{0}^{\infty} \frac{\left[\left(\sum_{i=1}^{m-1} \lambda_{i}\right) t\right]^{k} e^{-\left(\sum_{i=1}^{m-1} \lambda_{i}\right) t}}{k !} \int_{0}^{t} \frac{\left(\sum_{j=1}^{m-1} \mu_{j}\right) e^{-\left(\sum_{j=1}^{m-1} \mu_{j}\right) \tau}\left[\left(\sum_{j=1}^{m-1} \mu_{j}\right) \tau\right]^{n-1}}{(n-1) !} d \tau \\
& \cdot\left(\mu_{m}-\lambda_{m}\right) e^{-\left(\mu_{m}-\lambda_{m}\right) t} d t \\
= & \left(\sum_{j=1}^{m-1} \mu_{j}\right)\left(\mu_{m}-\lambda_{m}\right) \sum_{k=n}^{\infty} \int_{0}^{\infty} \int_{\tau}^{\infty} \frac{e^{-\left(\sum_{i=1}^{m-1} \lambda_{i}+\mu_{m}-\lambda_{m}\right) t}\left[\left(\sum_{i=1}^{m-1} \lambda_{i}\right) t\right]^{k}}{k !} d t \\
& \cdot \frac{e^{-\left(\sum_{j=1}^{m-1} \mu_{j}\right) \tau}\left[\left(\sum_{j=1}^{m-1} \mu_{j}\right) \tau\right]^{n-1}}{(n-1) !} d \tau
\end{aligned}
$$


In (4.25), let $t=\tau+u$ and simplify the equation

$$
\begin{aligned}
& \mathbb{P}\{\hat{M}(\hat{T}) \geq n\} \\
\leq & \left(\sum_{j=1}^{m-1} \mu_{j}\right)\left(\mu_{m}-\lambda_{m}\right) \sum_{k=n}^{\infty} \int_{0}^{\infty} \int_{0}^{\infty} \frac{e^{-\left(\sum_{i=1}^{m-1} \lambda_{i}+\mu_{m}-\lambda_{m}\right)(\tau+u)}\left[\left(\sum_{i=1}^{m-1} \lambda_{i}\right)(\tau+u)\right]^{k}}{k !} d u \\
& \cdot \frac{e^{-\left(\sum_{j=1}^{m-1} \mu_{j}\right) \tau}\left[\left(\sum_{j=1}^{m-1} \mu_{j}\right) \tau\right]^{n-1}}{(n-1) !} d \tau \\
= & \frac{\mu_{m}-\lambda_{m}}{\sum_{i=1}^{m-1} \lambda_{i}+\mu_{m}-\lambda_{m}}\left(\frac{\sum_{j=1}^{m-1} \mu_{j}}{\sum_{i=1}^{m-1} \lambda_{i}+\sum_{j=1}^{m-1} \mu_{j}+\mu_{m}-\lambda_{m}}\right)^{n} \\
& \sum_{k=n}^{\infty}\left(\frac{\sum_{i=1}^{m-1} \lambda_{i}}{\sum_{i=1}^{m-1} \lambda_{i}+\sum_{j=1}^{m-1} \mu_{j}+\mu_{m}-\lambda_{m}}\right)^{k} \\
& \cdot \sum_{l=0}^{k} \frac{(n-1+k-l) !}{(k-l) !(n-1) !}\left(\frac{\sum_{i=1}^{m-1} \lambda_{i}+\sum_{j=1}^{m-1} \mu_{j}+\mu_{m}-\lambda_{m}}{\sum_{i=1}^{m-1} \lambda_{i}+\mu_{m}-\lambda_{m}}\right)^{l}
\end{aligned}
$$

Now, let

$$
\begin{aligned}
& \alpha(k, l)=\frac{(n-1+k-l) !}{2^{k}(k-l) !(n-1) !} \\
& \beta=\frac{\sum_{i=1}^{m-1} \lambda_{i}+\sum_{j=1}^{m-1} \mu_{j}+\mu_{m}-\lambda_{m}}{\sum_{i=1}^{m-1} \lambda_{i}+\mu_{m}-\lambda_{m}}
\end{aligned}
$$

for $l=0,1, \cdots, k$. 
We have known that $\alpha(k, l)$ is maximized at $k=n+l-1$. Hence,

$$
\begin{aligned}
& \sum_{l=0}^{k} \frac{(n-1+k-l) !}{(k-l) !(n-1) !}\left(\frac{\sum_{i=1}^{m-1} \lambda_{i}+\sum_{j=1}^{m-1} \mu_{j}+\mu_{m}-\lambda_{m}}{\sum_{i=1}^{m-1} \lambda_{i}+\mu_{m}-\lambda_{m}}\right)^{l} \\
= & 2^{k} \sum_{l=0}^{k} \alpha(k, l) \beta^{l} \\
\leq & 2^{k-n} \sum_{l=0}^{k} \frac{2(2 n-2) !}{(n-1) !(n-1) !}\left(\frac{\beta}{2}\right)^{l} \\
\leq & 2^{k-n} \frac{2(2 n-2) !}{(n-1) !(n-1) !} \sum_{l=0}^{\infty}\left(\frac{\beta}{2}\right)^{l} \\
= & 2^{k-n} \frac{2(2 n-2) !}{(n-1) !(n-1) !} \frac{2}{2-\beta}
\end{aligned}
$$

From all the conclusions above, we can show that

$$
\begin{aligned}
& \mathbb{P}\{M(\hat{T}) \geq n\} \\
\leq & \frac{4\left(\mu_{m}-\lambda_{m}\right)}{\mu_{m}-\lambda_{m}-\left(\sum_{j=1}^{m-1} \mu_{j}\right)+\left(\sum_{i=1}^{m-1} \lambda_{i}\right)} \frac{(2 n-2) !}{2^{n}(n-1) !(n-1) !} \\
& \cdot\left(\frac{\sum_{j=1}^{m-1} \mu_{j}}{\sum_{i=1}^{m-1} \lambda_{i}+\sum_{j=1}^{m-1} \mu_{j}+\mu_{m}-\lambda_{m}}\right)^{n} \frac{\left(\frac{2\left(\sum_{i=1}^{m-1} \lambda_{i}\right)}{\sum_{i=1}^{m-1} \lambda_{i}+\sum_{j=1}^{m-1} \mu_{j}+\mu_{m}-\lambda_{m}}\right)^{n}}{1-\frac{2\left(\lambda_{1}+\lambda_{2}\right)}{\sum_{i=1}^{m-1} \lambda_{i}+\sum_{j=1}^{m-1} \mu_{j}+\mu_{m}-\lambda_{m}}}
\end{aligned}
$$

By Stirling's approximation, for large enough $n$,

$$
\begin{aligned}
\frac{(2 n) !}{n ! n !} & =\frac{\sqrt{4 \pi n}(2 n)^{2} n e^{-2 n}(1+O(1 / n))}{\left[\sqrt{2 \pi n}(n)^{n} e^{-n}(1+O(1 / n))\right]^{2}} \\
& \leq \frac{C_{2}}{\sqrt{n}} 4^{n}
\end{aligned}
$$

for some positive constant $C_{2}$. 
Therefore,

$$
\mathbb{P}\{\hat{M}(\hat{T}) \geq n\} \leq \frac{M_{4}}{\sqrt{n}}\left(\frac{4\left(\sum_{i=1}^{m-1} \lambda_{i}\right)\left(\sum_{j=1}^{m-1} \mu_{j}\right)}{\left(\sum_{i=1}^{m-1} \lambda_{i}+\sum_{j=1}^{m-1} \mu_{j}+\mu_{m}-\lambda_{m}\right)^{2}}\right)^{n}
$$

Where $M_{4}$ is given by combining the constant items in the equation which do not depend on $n$.

\section{Large Deviation Result for $\hat{M}(\hat{T})$ in Case 2}

At this case, it is easy to show that if we take Log and multiply $\frac{1}{n}$ on both bounds, the limit of them will be converge to the same value, that is,

$$
\log \left\{\frac{4\left(\sum_{i=1}^{m-1} \lambda_{i}\right)\left(\sum_{j=1}^{m-1} \mu_{j}\right)}{\left(\sum_{i=1}^{m-1} \lambda_{i}+\sum_{j=1}^{m-1} \mu_{j}+\mu_{m}-\lambda_{m}\right)^{2}}\right\}
$$

By the Squeezing Theorem,

$$
\lim _{n \rightarrow \infty} \frac{1}{n} \log \mathbb{P}\{\hat{M}(\hat{T}) \geq n\}=\log \left\{\frac{4\left(\sum_{i=1}^{m-1} \lambda_{i}\right)\left(\sum_{j=1}^{m-1} \mu_{j}\right)}{\left(\sum_{i=1}^{m-1} \lambda_{i}+\sum_{j=1}^{m-1} \mu_{j}+\mu_{m}-\lambda_{m}\right)^{2}}\right\}
$$

\subsubsection{Large Deviation Results for $\hat{M}(\hat{T})$}

Combining the results in Section 4.2.1 and Section 4.2.2,we obtain that 
Theorem 4.1

$$
\begin{aligned}
& \lim _{n \rightarrow \infty} \frac{1}{n} \log \mathbb{P}\{\hat{M}(\hat{T}) \geq n\} \\
= & \begin{cases}\log \left\{\frac{\sum_{i=1}^{m-1} \lambda_{i}}{\sum_{i=1}^{m-1} \lambda_{i}+\mu_{m}-\lambda_{m}}\right\}, & \text { if } \sum_{i=1}^{m-1}\left(\mu_{i}-\lambda_{i}\right) \geq \mu_{m}-\lambda_{m}\end{cases} \\
\log \left\{\frac{4\left(\sum_{i=1}^{m-1} \lambda_{i}\right)\left(\sum_{j=1}^{m-1} \mu_{j}\right)}{\left(\sum_{i=1}^{m-1} \lambda_{i}+\sum_{j=1}^{m-1} \mu_{j}+\mu_{m}-\lambda_{m}\right)^{2}}\right\}, & \text { if } \sum_{i=1}^{m-1}\left(\mu_{i}-\lambda_{i}\right)<\mu_{m}-\lambda_{m}
\end{aligned}
$$

\section{Lemma 4.1}

$$
\begin{aligned}
& \lim _{n \rightarrow \infty} \frac{1}{n} \log \mathbb{P}\{\hat{M}(\hat{T}) \geq n \bigcap \hat{q}(\hat{T})=0\} \\
& \geq \begin{cases}\log \left\{\frac{\sum_{i=1}^{m-1} \lambda_{i}}{\sum_{i=1}^{m-1} \lambda_{i}+\mu_{m}-\lambda_{m}}\right\}, & \text { if } \sum_{i=1}^{m-1}\left(\mu_{i}-\lambda_{i}\right) \geq \mu_{m}-\lambda_{m}\end{cases} \\
& \log \begin{cases}4\left(\sum_{i=1}^{m-1} \lambda_{i}\right)\left(\sum_{j=1}^{m-1} \mu_{j}\right) \\
\left.\frac{\left(\sum_{i=1}^{m-1} \lambda_{i}+\sum_{j=1}^{m-1} \mu_{j}+\mu_{m}-\lambda_{m}\right)^{2}}{}\right\}, & \text { if } \sum_{i=1}^{m-1}\left(\mu_{i}-\lambda_{i}\right)<\mu_{m}-\lambda_{m}\end{cases}
\end{aligned}
$$

Note that in the lemma above, the event $\{\hat{q}(\hat{T})=0\}$ is equivalent to the event $\left\{\bigcap_{i=1}^{m-1} \hat{q}_{i}(\hat{T})=0\right\}$.

\subsection{Conclusion}

In this section, we will combine the results of previous two sections and prove the main theorem. As in the beginning of this chapter, we have assumed $\lambda_{1}=\lambda_{2}=$ $\cdots=\lambda_{m}$ and $\mu_{1}=\mu_{2}=\cdots=\mu_{m}$. 
Theorem 4.2 (Main Result 2)

$$
\lim _{n \rightarrow \infty} \frac{1}{n} \log \mathbb{P}\left\{q^{r}(t) \geq n\right\}=\log \left\{\frac{(m-1) \lambda_{1}}{(m-2) \lambda_{1}+\mu_{1}}\right\}
$$

Proof. Without th e loss of generality, we just show the case when $m$ is odd. If $m$ is even, using the similar analysis, we can get the same concussion.

Start with (4.10) and the density function (4.8)

$$
\begin{aligned}
& \int_{0+}^{\infty} \mathbb{P}\left\{M_{(1)}(t, s) \geq n \mid \bigcap_{j=2}^{m} W_{j}(t)<s\right\} f_{W_{1} \mid \bigcap_{j=2}^{m} W_{1}>W_{j}}(s) d s \\
\geq & \int_{0+}^{\infty} \mathbb{P}\left\{M_{(1)}(s) \geq n, \bigcap_{j=2}^{m} q_{j}(t)=0\right\} f_{W_{1} \mid \bigcap_{j=2}^{m} W_{1}>W_{j}}(s) d s \\
= & A_{1} \int_{0}^{\infty} \mathbb{P}\left\{M_{(1)}(s) \geq n, \bigcap_{j=2}^{m} q_{j}(t)=0\right\} e^{-\left(\mu_{1}-\lambda_{1}\right) x} d s \\
& -\sum_{k=2}^{m} \int_{0}^{\infty} A_{k} \mathbb{P}\left\{M_{(1)}(s) \geq n, \bigcap_{j=2}^{m} q_{j}(t)=0\right\} e^{-\left(\mu_{1}-\lambda_{1}+\mu_{k}-\lambda_{k}\right) x} d s \\
& +\sum_{p=2}^{m} \sum_{q=p+1}^{m} \int_{0}^{\infty} A_{p q} \mathbb{P}\left\{M_{(1)}(s) \geq n, \bigcap_{j=2}^{m} q_{j}(t)=0\right\} e^{-\left(\mu_{1}-\lambda_{1}+\mu_{p}-\lambda_{p}+\mu_{q}-\lambda_{q}\right) x} d s \\
& +\cdots \\
& +A_{12 \cdots m} \mathbb{P}\left\{M_{(1)}(s) \geq n, \bigcap_{j=2}^{m} q_{j}(t)=0\right\} e^{-\left(\mu_{1}-\lambda_{1}+\mu_{2}-\lambda_{2}+\cdots+\mu_{m}-\lambda_{m}\right) x} d s
\end{aligned}
$$

where $A_{1}, A_{2}, \cdots, A_{m}, A_{23}, \cdots$ are positive constants. There are $2^{m-1}$ items in the inequality above. Next we will find the maximum value of these $2^{m-1}$ items. 
For the first item $A_{1} \int_{0}^{\infty} \mathbb{P}\left\{M_{(1)}(s) \geq n, \bigcap_{j=2}^{m} q_{j}(t)=0\right\} e^{-\left(\mu_{1}-\lambda_{1}\right) x} d s$, since $\sum_{i=2}^{m}\left(\mu_{i}-\right.$ $\left.\lambda_{i}\right) \geq \mu_{1}-\lambda_{1}$ by Lemma (4.1), it yields

$$
\begin{aligned}
& \lim _{n \rightarrow \infty} \frac{1}{n} \log \int_{0}^{\infty} \mathbb{P}\left\{M_{(1)}(s) \geq n, \bigcap_{j=2}^{m} q_{j}(t)=0\right\} e^{-\left(\mu_{1}-\lambda_{1}\right) x} d s \\
\geq & \log \left\{\frac{\sum_{i=2}^{m} \lambda_{i}}{\sum_{i=2}^{m} \lambda_{i}+\mu_{1}-\lambda_{1}}\right\} \\
= & \log \left\{\frac{(m-1) \lambda_{1}}{(m-2) \lambda_{1}+\mu_{1}}\right\}
\end{aligned}
$$

For the type of $\int_{0}^{\infty} A_{k} \mathbb{P}\left\{M_{(1)}(s) \geq n, \bigcap_{j=2}^{m} q_{j}(t)=0\right\} e^{-\left(\mu_{1}-\lambda_{1}+\mu_{k}-\lambda_{k}\right) x}$, where $k=$ $2,3, \cdots, m$. Since all the $\lambda_{i}$ are equal and $\mu_{i}$ are equal, all these type will have the same lower bound. Hence, we just need solve when $k=2$. By Lemma (4.1), we have

$$
\begin{aligned}
& \lim _{n \rightarrow \infty} \frac{1}{n} \log \int_{0}^{\infty} \mathbb{P}\left\{M_{(1)}(s) \geq n, \bigcap_{j=2}^{m} q_{j}(t)=0\right\} e^{-\left(\mu_{1}-\lambda_{1}+\mu_{2}-\lambda_{2}\right) x} d s \\
\geq & \log \left\{\frac{\sum_{i=2}^{m} \lambda_{i}}{\sum_{i=2}^{m} \lambda_{i}+\mu_{1}-\lambda_{1}+\mu_{2}-\lambda_{2}}\right\} \\
= & \log \left\{\frac{(m-1) \lambda_{1}}{(m-3) \lambda_{1}+2 \mu_{1}}\right\}
\end{aligned}
$$

The inequality is true, because $\sum_{i=2}^{m}\left(\mu_{i}-\lambda_{i}\right) \geq \mu_{1}-\lambda_{1}+\mu_{2}-\lambda_{2}$.

Similarly, for the type $\int_{0}^{\infty} A_{p q} \mathbb{P}\left\{M_{(1)}(s) \geq n, \bigcap_{j=2}^{m} q_{j}(t)=0\right\} e^{-\left(\mu_{1}-\lambda_{1}+\mu_{p}-\lambda_{p}+\mu_{q}-\lambda_{q}\right) x} d s$, where $p=2,3, \cdots, m$ and $q=p+1, \cdots, m$, we just compute the case of 
$p=2, q=3$.

$$
\begin{aligned}
& \lim _{n \rightarrow \infty} \frac{1}{n} \log \int_{0}^{\infty} \mathbb{P}\left\{M_{(1)}(s) \geq n, \bigcap_{j=2}^{m} q_{j}(t)=0\right\} e^{-\left(\mu_{1}-\lambda_{1}+\mu_{2}-\lambda_{2}+\mu_{3}-\lambda_{3}\right) x} d s \\
\geq & \log \left\{\frac{\sum_{i=2}^{m} \lambda_{i}}{\sum_{i=2}^{m} \lambda_{i}+\mu_{1}-\lambda_{1}+\mu_{2}-\lambda_{2}+\mu_{3}-\lambda_{3}}\right\} \\
= & \log \left\{\frac{(m-1) \lambda_{1}}{(m-4) \lambda_{1}+3 \mu_{1}}\right\}
\end{aligned}
$$

Except $A_{12 \cdots m} \mathbb{P}\left\{M_{(1)}(s) \geq n, \bigcap_{j=2}^{m} q_{j}(t)=0\right\} e^{-\left(\mu_{1}-\lambda_{1}+\mu_{2}-\lambda_{2}+\cdots+\mu_{m}-\lambda_{m}\right) x} d s$, we always have $\sum_{i=2}^{m}\left(\mu_{i}-\lambda_{i}\right)$ is greater or equal to the power of exponent, so we can use the first case of Lemma (4.1). It is easy to check the maximum value of these $2^{m-1}-1$ items is $\log \left\{\frac{(m-1) \lambda_{1}}{(m-2) \lambda_{1}+\mu_{1}}\right\}$, according to the fact that $u_{1}>\lambda_{1}$.

In the last item, we have $\sum_{i=1}^{m-1}\left(\mu_{i}-\lambda_{i}\right)<\mu_{1}-\lambda_{1}+\cdots+\mu_{m}-\lambda_{m}$

$$
\begin{aligned}
& \lim _{n \rightarrow \infty} \frac{1}{n} \log \int_{0}^{\infty} \mathbb{P}\left\{M_{(1)}(s) \geq n, \bigcap_{j=2}^{m} q_{j}(t)=0\right\} e^{-\left(\mu_{1}-\lambda_{1}+\mu_{2}-\lambda_{2}+\cdots+\mu_{m}-\lambda_{m}\right) x} d s \\
\geq & \log \left\{\frac{4\left(\sum_{i=2}^{m} \lambda_{i}\right)\left(\sum_{j=2}^{m} \mu_{j}\right)}{\left[\sum_{i=2}^{m} \lambda_{i}+\sum_{j=2}^{m} \mu_{j}+\sum_{k-1}^{m}\left(\mu_{k}-\lambda_{k}\right)\right]^{2}}\right\} \\
= & \log \left\{\frac{4(m-1)^{2} \lambda_{1} \mu_{1}}{\left[(2 m-1) \mu_{1}-\lambda_{1}\right]^{2}}\right\}
\end{aligned}
$$

To compare $\frac{(m-1) \lambda_{1}}{(m-2) \lambda_{1}+\mu_{1}}$ and $\frac{4(m-1)^{2} \lambda_{1} \mu_{1}}{\left[(2 m-1) \mu_{1}-\lambda_{1}\right]^{2}}$.

$$
\frac{(m-1) \lambda_{1}}{(m-2) \lambda_{1}+\mu_{1}}=\frac{4(m-1)^{2} \lambda_{1} \mu_{1}}{4(m-1) \mu_{1} \cdot\left[(m-2) \lambda_{1}+\mu_{1}\right]}
$$

Now, these two fractions have the same numerator, then we just need compare their denominator. 


$$
\begin{aligned}
& {\left[(2 m-1) \mu_{1}-\lambda_{1}\right]^{2}-4(m-1) \mu_{1} \cdot\left[(m-2) \lambda_{1}+\mu_{1}\right] } \\
= & \left(4 m^{2}-8 m+5\right) \mu_{1}^{2}-\left(4 m^{2}-16 m+14\right) \lambda_{1} \mu_{1}+\lambda_{1}^{2} \\
= & (2 m-2)^{2} \mu_{1}^{2}+(2 m-4)^{2} \lambda_{1} \mu_{1}+\left(\mu_{1}-\lambda_{1}\right)^{2} \geq 0
\end{aligned}
$$

Thus, the maximum value of these $2^{m-1}$ items is $\log \left\{\frac{(m-1) \lambda_{1}}{(m-2) \lambda_{1}+\mu_{1}}\right\}$.

Therefore,

$$
\begin{aligned}
& \lim _{n \rightarrow \infty} \frac{1}{n} \log \left\{\int_{0+}^{\infty} \mathbb{P}\left\{M_{(1)}(t, s) \geq n \mid \bigcap_{j=2}^{m} W_{j}(t)<s\right\} f_{W_{1} \mid \cap_{j=2}^{m} W_{1}>W_{j}}(s) d s\right\} \\
\geq & \log \left\{\frac{(m-1) \lambda_{1}}{(m-2) \lambda_{1}+\mu_{1}}\right\}
\end{aligned}
$$

Using similar arguments and by Theorem (4.1), we obtain,

$$
\begin{aligned}
& \lim _{n \rightarrow \infty} \frac{1}{n} \log \left\{\int_{0+}^{\infty} \mathbb{P}\left\{M_{(1)}(t, s) \geq n\right\} f_{W_{1} \mid \cap_{j=2}^{m} W_{1}>W_{j}}(s) d s\right\} \\
\leq & \log \left\{\frac{(m-1) \lambda_{1}}{(m-2) \lambda_{1}+\mu_{1}}\right\}
\end{aligned}
$$

As we discussed in the previous chapter, it is an upper bound of

$$
\lim _{n \rightarrow \infty} \frac{1}{n} \log \left\{\int_{0+}^{\infty} \mathbb{P}\left\{M_{(1)}(t, s) \geq n \mid \bigcap_{j=2}^{m} W_{j}(t)<s\right\} f_{W_{1} \mid \cap_{j=2}^{m} W_{1}>W_{j}}(s) d s\right\}
$$

Thus both upper and lower bounds converge to the same value, by the Squeezing Theorem and (4.3),

$$
\lim _{n \rightarrow \infty} \frac{1}{n} \log \left\{\mathbb{P}\left\{M_{(1)}(t, s) \geq n \mid \bigcap_{j=2}^{m} W_{1}>W_{j}(t)\right\}\right\}=\log \left\{\frac{(m-1) \lambda_{1}}{(m-2) \lambda_{1}+\mu_{1}}\right\}
$$


By symmetry and the conditions that $\lambda_{1}=\lambda_{2}=\cdots \lambda_{m}, \mu_{1}=\mu_{2}=\cdots \mu_{m}$, we can easily show that

$$
\begin{aligned}
& \lim _{n \rightarrow \infty} \frac{1}{n} \log \left\{\mathbb{P}\left\{M_{(i)}\left(t, W_{*}(t)\right) \geq n \mid \bigcap_{\substack{j=1 \\
j \neq i}}^{m} W_{i}(t)>W_{j}(t)\right\}\right\} \\
= & \log \left\{\frac{(m-1) \lambda_{1}}{(m-2) \lambda_{1}+\mu_{1}}\right\}
\end{aligned}
$$

for all $i=2,3, \cdots, m$.

Combining all the result above and function (4.2), we can get Theorem 4.2 


\section{Bibliography}

[1] A. Jean-Marie and L. Gün. Parallel queues with resequencing. Journal of the Association for Computing Machinery, Vol. 40, No. 5, pages 1188C 1208, November 1993. [9] F. Kamoun, L. Kleinrock,

[2] Ayalvadi Ganesh, Neil O'Connell and Damon Wischik. Big Queues. SpringerVerlag, 2004.

[3] Chang, C.-S., Chao, X.-L., and Pinedo, M. A note on queues with Bernoulli routing. In Proceedinds of the 29th Conference on Decision and Control (Hawaii, Dec.), 1990.

[4] Frank den Hollander. Large Deviations. Amer. Math. Soc., 2000.

[5] Gün, L., Jean-Marie, A., Makowski, M., and Tedijanto, T. Convexity results for parallel queues with Bernoulli routing. Univ. Maryland, College Park, ISR Tech. Rep., 1990.

[6] Han Y, Makowski A. Resequencing delays under multipath routing C Asymptotics in a simple queueing model. In INFOCOM, 2006. 
[7] J. W. Cohen. The Single Server Queue. North-Holland Publishing Company, Revised edition, 1982.

[8] Leonard Kleinrock. Queue Systems, Volume I: Theory. Jonh Wiley \& Sons, 1975.

[9] Marc Lelarge. Packet reordering in networks with heavy-tailed delays. Mathematical Methods of Operations Research, Vol. 67, No. 2, pages 341-371, 2008.

[10] N. Gogate and S. S. Panwar. On a resequencing model for high speed networks. In Proceedings of INFOCOM '94, pages 40-47, Toronto, Canada, June 1994.

[11] J. George Shanthikumar. Stochastic majorization of random variables with proportional equilibrium rates. Adv. Appl. Prob. 19 (Mar. 1987), 854-872.

[12] Sheldon M. Ross. Introduction to Probability Models. Elsevisr, Eighth Edition, 2004.

[13] Ye Xia and David Tse. On the large deviation of resequencing queue size: 2-M/M/1 case. In INFOCOM 2004. Twenty-third AnnualJoint Conference of the IEEE Computer and Communications Societies, Vol. 4, pages 23742382, March 2004.

[14] Z. Rosberg and N. Shacham. Resequencing delay and buffer occupancy under the selective-repeat ARQ. IEEE Transactions on Information Theory, Vol. 35, No. 1, pages 166C172, January 1989. 
[15] Z. Rosberg and M. Sidi. Selective-repeat ARQ: the joint distribution of the transmitter and the receiver resequencing buffer occupancies. IEEE Transactions on Communications, Vol. 38, No. 9, pages 1430C1438, September 1990 


\section{Index}

$M / M / 1$ queue, 13

automatic repeat-request, 9

Central Limit Theorem, 14, 35

disordering network, 9, 16

ergodic, 14

First-In-First-Out, 9

Large deviation, $14,15,18,58$

queue size, 15

Multilink Protocol, 3

Point to Point, 3

Poisson

process, 11-14, 16, 29, 66

Split Property, 12, 16

re-sequencing

buffer/queue, $9,10,17$

buffer/queue size, 10 delay, 10

Squeezing Theorem, 38, 49, 55, 68, 74, 79

Stability Condition, 13

stationary, $15,18,28,30,58,65$

increment, 11

probability distribution, 14,15

Stirling's approximation, 36, 40, 46, 68,

69,73

time reversible, 14 